\title{
TEXTGESCHICHTLICHE BEOBACHTUNGEN AM SCHLUSSTEXT VON TOTENBUCHSPRUCH 146
}

\author{
VON \\ URSULA VERHOEVEN
}

Die Tradierungsgeschichte des Totenbuches nach dem Neuen Reich bzw. der 21. Dynastie ist ein Forschungsbereich, dessen Bearbeitung noch in den Anfängen steckt ${ }^{1}$. Im Zuge der Bearbeitung des Kölner Totenbuches der Iahtesnach aus der 26. Dynastie ${ }^{2}$ zeigten sich bei Vergleichen mit anderen Quellen der Spätzeit und der Ptolemäerepoche manche Einblicke in die textgeschichtliche Entwicklung, aus der die sogenannte «saitische Rezension» des Totenbuches hervorgegangen ist. Als Beispiel sei eine relativ kurze Texteinheit herausgegriffen, der Schlußtext von Tb 146.

Dieser Text, der der Nennung und Abbildung der 21 Tore von Tb 146 folgt, findet sich anscheinend erstmals in der 21. Dynastie ${ }^{3}$ und lebt bis in ptolemäische Zeit fort ${ }^{4}$. Von den publizierten Totenbüchern enthalten ihn die Totenbücher von Gatseschni ${ }^{5}(\mathbf{C g})$ und Nesitanebetascheru ${ }^{6}$ (Ec), nicht aber die Papyri von Kamara und Nesichonsu ${ }^{7}$. Der persisch-ptolemäische pOIM $9787^{8}$ (R) führt ihn ebenso wie die ptolemäischen Quellen pTurin $1791^{9}(\mathbf{T})$ und pLeiden T $16^{10}(\mathbf{L})$, beim gleichzeitigen pMilbank ${ }^{11}$ fehlt er jedoch wieder.

Von der großen Anzahl später Totenbücher aus der Zeit zwischen diesen Epochen sind diejenigen Quellen, die mit ziemlicher Sicherheit in die saitische Dynastie datiert werden können, äußerst selten ${ }^{12}$ :

\footnotetext{
1 Einen wichtigen Beitrag lieferte kürzlich U. Rössler-Köhler, «Totenbuch-Tradierung nach dem NR und bis Spätzeitbeginn», in: U. Verhoeven, E, Graefe, Hgg., Religion und Philosophie im Alten Ägypten, Festgabe für Ph. Derchain, (OLA 39, 1991), S. $277 \mathrm{ff}$.

${ }^{2}$ Verhoeven, Das saitische Totenbuch der Iahtesnacht, P. Colon. Aeg. 10207, mit einem Beitrag von P. Dils, Papyrologische Texte und Abhandlungen 3 Bde., im Druck.

3 Ich danke Frau I. Munro, Bonn, für die Durchsicht der ihr zugänglichen Tb-Manuskripte der 18.-19. Dyn., die bezüglich der Suche nach diesem Text negativ verlief.

${ }^{4}$ Graefe, Der "Sonnenaufgang zwischen den Pylontürmen», in: OLP 14 (1983), 58f. schrieb bezüglich der Quelle T: «Eine ältere (Handschrift), die diesen (Text, d.h. die Nachschrift zu Tb 146) enthält, habe ich bisher nicht finden können».

${ }^{5}$ Naville, Papyrus funéraires de la XXIe dynastie, II, (1914), Tf. 41-2.

6 Budge, The Greenfield Papyrus in the British Museum, (1912), Tf. 52.

7 Vgl. die Publikation von Naville, Papyrus funéraires de la XXIe dynastie, I, (1912).

8 "pRyerson", Allen, The Egyptian Book of the Dead, Documents in the Oriental Institute Museum of Chicago, (OIP 82, 1960), Tf. 43, Kol. 131.

9 Abschrift: Lepsius, Das Todtenbuch der Ägypter nach dem hieroglyphischen Papyrus in Turin, (1842, Nachdruck Osnabrück 1969), Tf. 65-67; Fotos: De Rachewiltz, Il Libro dei Morti degli antichi Egiziani, (1958), 76. 80.

10 Leemans, Monuments égyptiens du Musée d'antiquités des Pays-Bas à Leide, III, (1867), Tf. 34.

11 Veröffentlicht von Allen, The Egyptian Book of the Dead, passim.

12 Ich danke St. Quirke für den Austausch von Informationen.
} 
- pNespasef (Nes.),

— pColon. Aeg. $10207^{18}$

- pVatikan $\mathrm{I}^{20}$

- pOIM 5739 ehem. Sammlung Brunner ${ }^{13}$ und Musée de la Vieille Charité (früher Musée Borély), Marseille, Inv. Nr. 291.

Besitzer: Monthpriester Nespasef, Sohn des Besenmut und der Habenesamun ${ }^{14}$, Ende 25., Anfang 26. Dynastie (Psammetich I.)

— pDeir el Bahari F. $5594^{15}$

Besitzer: Irtirutjau (Irti.), Sohn des Djedchonsuiufanch und der Tascheritenaset ${ }^{16}$. Er gehört wohl wie Nespasef zur Besenmut-Familie ${ }^{17}$ und ist zu Anfang bis Mitte der 26. Dynastie zu datieren.

Besitzerin: Iahtesnacht (Iah.), Tochter des Pascherienawy und der Tadiu, nach dem Stil des Sarges aus der mittleren Saitenzeit (ca. 600 v. Chr.) ${ }^{19}$.

Besitzer: Psammetich, Sohn der Sebarechit, späte 26. Dynastie $^{21}$.

Besitzer: Anchpefheri, Sohn des Tjamabanacht, besitzt paläographisch und im Textbestand große Ähnlichkeiten mit pColon. Aeg. $10207^{22}$ und weist außerdem aufgrund der Namensformen in die Saitenzeit.

Bei den beiden ersten Quellen sind nur Teile des Totenbuches erhalten, der Schlußtext von Spruch 146 (von Allen als «BD $146 \mathrm{w}$ » bezeichnet ${ }^{23}$ ) findet sich aber bei beiden, bei Irti. allerdings sehr fragmentarisch. Im Kölner $\mathrm{Tb}$ (Iah.) ist dieser Text vollständig überliefert. Die Fragmente des Chicagoer Exemplars bieten den Spruch nicht, der Text des Vatikan-Totenbuches kann aufgrund der unzureichenden Veröffentlichung nicht herangezogen werden.

Ein weiteres fragmentarisch erhaltenes Totenbuch zeigt diesen Schlußtext, der pTübingen 2000 eines gewissen Pefiu ${ }^{24}$ (Pef.), der in die «frühe Spätzeit» datiert wird. Er ist nach

13 Veröffentlicht: E. Brunner-Traut-H. Brunner, Die ägyptische Sammlung der Universität Tübingen, (1981), S. 293 mit Tf. 126. 127. Ich danke Herrn Professor Dr. H. Brunner für die Übersendung seiner Fotos dieses Papyrus, durch die die Abschrift erleichtert wurde. Auch dieser Teil des Papyrus des Nespasef befindet sich jetzt im Musée de la Vieille Charité in Marseille.

14 Vittmann, Priester und Beamte im Theben der Spätzeit, (Beiträge zur Ägyptologie 1, 1978), S. 23.

15 Dabrowska-Smektala, "Fragment of Hieratic Papyrus of $I r . t y-r w-t 3 w$ », in: BIFAO 66 (1966), $183 \mathrm{ff}$. mit Tf. 47.

16 Zur Familie und dem Sarg desselben Mannes (BIFAO 66, Tf. 35 f.) vgl. Vittmann, Priester und Beamte, S. 24.

$17 \mathrm{Vgl}$. auch Bierbrier, The Late New Kingdom in Egypt (c. 1300-664 B.C.), (1975), S. 92, chart 22 (Iretertja).

18 Siehe Anm. 2.

19 Dils, in: Verhoeven, Das saitische Totenbuch, S. $11 \mathrm{f}$.

20 Marucchi, Il grande papiro egizio della Biblioteca Vaticana, (1888).

21 Yoyotte, "La sépulture du père divin Psamétik, fils de la dame Sbarekhy», in: BSFE 60 (1971), $21 \mathrm{f}$.

22 Vgl. dazu Verhoeven, Das saitische Totenbuch, S. 370.

23 Allen, The Egyptian Book of the Dead, S. 246 f. (pRyerson); Ders. (†), The Book of the Dead or Going Forth by Day, (SAOC 37, 1974), S. $136 \mathrm{f}$.

${ }^{24}$ Veröffentlicht bei E. Brunner-Traut-H. Brunner, Die ägyptische Sammlung, S. 294, Tf. 107; K. Buroh et al., 
seiner formalen Gestaltung und der Art seiner Vignetten dem Kölner Totenbuch (und den anderen der 26. Dynastie) sehr ähnlich ${ }^{25}$, und es wird sich zeigen, daß er aufgrund seiner textlichen Gestaltung an den r.nfang der 26. Dynastie (in die Nähe von Nes.) zu datieren ist.

Anhand der vollständig erhaltenen Quellen Cg, Ec, Iah., R, T und L war es möglich, die fragmentarischen Texte Nes., Pef. und Irti. einzuhängen. Die synoptische Textzusammenstellung der aufgewählten neun Textzeugen (S. 179-94) läßt eine textgeschichtliche Gruppierung ${ }^{26}$ erkennen, die im folgenden näher zu erläutern sein wird.

Der Text fällt durch seine Gliederung in Strophen auf, die - bis auf Einleitung und Schluß - mit den Worten jy. $n=j \operatorname{mjn} h r s b 3 n \ldots$ beginnen ${ }^{27}$. Der Textbestand ist in allen benutzten Quellen fast gleich, die Reihenfolge der «Strophen» variiert jedoch. Sie werden hier, basierend auf der Reihenfolge der ältesten Texte $\mathrm{Cg}$ und Ec, mit den Buchstaben A$\mathrm{R}$ gekennzeichnet ${ }^{28}$. Auf eine erneute Übersetzung wird an dieser Stelle verzichtet ${ }^{29}$, der Textbestand lautet wie folgt (Varianten, die bei mindestens zwei Textzeugen belegt sind, sind hinter einem Schrägstrich aufgeführt, ebenso bezeugte Textergänzungen stehen in Klammern):

$$
\begin{aligned}
& \text { A } \quad W \operatorname{sjr} N . \underline{d} \boldsymbol{d}=\boldsymbol{f} \\
& j n k w n h . w j 3 . t=f \text { prj. } w m \text { wrr. } t^{30} \\
& j y . n=j \operatorname{smn} . n=j \underline{h} . t m 3 b \underline{d} w \\
& w p . n=j w 3 . t m R 3-s t 3 w^{31} \\
& s n d m . n=j m n . t \text { n.t Wsjr }{ }^{32} \\
& \text { B jy.n=j mjn hr sb3 njmh.t } \\
& \text { jmn (.w) jr } n=j w 3 . t
\end{aligned}
$$

Hieroglyphenschrift und Totenbuch. Die Papyri der Ägyptischen Sammlung der Universität Tübingen, (Ausstellungskataloge der Universität Tübingen Nr. 18, 1985), Umschlagbild und S. 51 ff. Zur Textrekonstruktion der Tore von Spruch 147 und weiteren Fragmenten dieses Totenbuches vgl. Verhoeven, «Das einzige bekannte Beispiel seiner Art», in: D. Mendel, U. Claudi, Hgg., Ägypten im afroorientalischen Kontext, Gs P. Behrens, Afrikanistische Arbeitspapiere, Sondernr. 1991, (1992), S. $405-10$,

25 Vgl. dazu Verhoeven, Das saitische Totenbuch, S. $43 \mathrm{f}$.

26 Von einem "Stemma" soll hier absichtlich nicht gesprochen werden, da die Anzahl und die zeitliche Entfernung der einzelnen Textzeugen eine nutzbringende Rekonstruktion dieser Form m.E. nicht erlauben.

${ }^{27}$ Ausnahme ist Abschnitt I, siehe dazu unten.

${ }_{28}$ Die von Allen anhand von R aufgestellte Zählung P, S 1-16, T wurde von ihm ebenfalls bezüglich Ec benutzt (vgl. die nächste Anm.) und mußte dabei umgestellt werden. Sie eignet sich daher m.E nicht für die hier vorgenommene textgeschichtliche Betrachtung, die ja von der Reihenfolge bei Ec ausgeht.

29 Übersetzung von EC: Allen, The Book of the Dead or Going Forth by Day, S. 136 f.; Irti.: Dabrowska-Smektala, in: BIFAO 66 (1966), 188 f.; Iah.: Verhoeven, Das saitische Totenbuch, S. 295-8; R: Allen, The Egyptian Book of the Dead S. $246 \mathrm{f}$.

30 So erst seit Pef. Cg. hat Spatien und unverständliche Restzeichen, Ec läßt die Passage bis auf wn aus.

${ }^{31} \mathrm{Cg}$ fügt ein überflüssiges $p w$ jmn.w ein, das eventuell eine Vorwegnahme aus der folgenden Strophe ist.

32 Parallelen zu dieser Strophe finden sich in $\mathrm{Tb} 117$ und 147, 1. und 3. Tor. 
jnk $H r n \underline{d}(h r) j t=f s 33 s t j w^{c} n W s j r$

$j y . n=j d r . n=j \underline{d h^{c 33}} h r$ Wsjr

C $j y . n=j \operatorname{mjn} h r s b 3 n h b(s)$

$h d n^{34}$ gmh.t jr $n=j w 3 . t$

jnk $\mathrm{Hr}$ nd $(\mathrm{hr}) \mathrm{jt}=\mathrm{f}$

$j y . n=j n h m . n=j 3 h 3 m^{-c} j r w s w$

D $j y . n=j \operatorname{mjn} h r s b 3 n$ (jmn.tt (ky $\underline{d} d) s b 3 n) d 3 t$

$n b(. w n d 3 . t) j r n=j w 3 . t$

jnk $\mathrm{Hr} n \underline{d}(\underline{h r}) j t=f$

$j y . n=j h s r . n=j \underline{d} w(n b) h r / j r j j t=j W s j r^{35}$

$b h n . n=j h f t j=f(s h r . n=j h f t j w=f)^{36}$

hr tz pwy n Ndy.t (hrw) pwy n psš $c_{3}$

E $\quad j y . n=j \operatorname{mjn} h r s b 3 n$ msms

$n b . w h 3 w . t^{37} j r n=j w 3 . t$

jnk $H r s 3 W s j r m w . t=j 3 s . t h r(j r . t n=j) m k . t=j$

$j y . n=j j n j . n=j c_{n h} w 3 s n j t=j W s j r^{38}$

$\mathbf{F} \quad j y . n=j \operatorname{mjn} h r s b 3 n q 3$

nb.w $n$ h. $\mathrm{j}$ j $n=j$ w3.t

jnk Hr s3 Wsjr (jw $\left.w^{c} n\right) W n-n f r\left(m 3^{c}-h r w\right)$

$j y . n=j(\mathrm{mjn}) \underline{h r} \check{s} z p w b 3(n=j) k k w$

G $j y . n=j \operatorname{mjn} h r$ sb3 $n \underline{t h n} . t$

$j m j . w$ tnn.t jr $n=j w 3 . t$

jnk nhh jmj h3.t wj3 (n Rc)

$j y . n=j r$ stp-s3 $h r$ Wsjr

$\mathbf{H} j y . n=j \operatorname{mjn} h r \operatorname{sb} 3 n h 3 y$

$n b . w$ h3y jr $n=j$ w3.t

jnk $\mathrm{Hr} s 3 \mathrm{mr}=f\left(m 3^{c}-h r w\right)$

I $\quad j y . n=j \operatorname{mjn}$ hr $s b 3 n \underline{t p h} . t$

rh. kwj sšt3.w ntj jm = st

$\mathbf{K} \quad j y . n=j \operatorname{mjn} c_{3} / d j m$ njw.t wr.t

jnk $R^{c} m d w 3$

$d j=j \underline{t} 3 w n{ }^{c} n h n(j t=j) W_{s j r}$

${ }^{33}$ Die Quellen zeigen hier sehr unterschiedliche Versionen. Das seltene Wort $d h^{c}$ wurde anscheinend mißverstanden.

34 So die Quellen bis Iah., danach unterschiedliche Auffassungen.

35 Vgl. die Passage im Text des 3. Tores von Tb 147 (Iah.): jw. $n=j d r . n=j \underline{d w} h r$ Wsjr.

${ }^{36}$ Mit Sicherheit nur bei Ec und L. Vgl. die Parallele im Schlußtext von Tb 145: shr. $n=j$ hftj.w=f nb.w.

37 Ebenfalls im Schlußtext von Tb 145 erwähnt.

${ }^{38} \mathrm{~Tb}$ 147, 4. Tor: $j n j . n=j c_{n h}$; Tb 145, Schlußtext: $j w . n=j d j=j{ }_{n h} n j t=j W_{s j r}$. 
L $\quad j y . n=j \operatorname{mjn} h r s b 3 n q m d y . t / q 3 y . t(k y \underline{d d})$ «Skorpion»

rh.kwj sw hrw

M jy. $n=j \operatorname{mjn} h r s b 3 n n b . w \underline{H} r^{-c} h 3$

( $\left.h n^{c}\right) s r . w n . w n b r-d r j r n=j w 3 . t$

$\mathbf{N} \quad j y . n=j \operatorname{mjn} h r s b 3 n$ wr.t/sd.t $\ldots / h^{c} p j^{39}$

$m s . w k k w$ jr $n=j w 3 . t$

O jy. $n=j \operatorname{mjn} h r s b 3 n h k 3 w$

h3 $n$ khb ntj $r g s=f j r n=j w 3 . t$

$\mathbf{P} \quad j y . n=j \operatorname{mjn} h r s b 3 n m r-g r$

$s g 3 \check{s y} . t /(k y \underline{d d})$ sqryw.t hntj $m k \cdot t=j$

(hr) jy $n=k$ hr shn jr.t $\mathrm{Hr}$

( hrj.w njw.t jr $n=j w 3 . t$

$h m \operatorname{sšm}(=j) t w=j$

Q jy.n $=\mathrm{j} \operatorname{mjn} \mathrm{h} r \operatorname{sb} 3 n$ hpt $w r^{40}$ )

hrj.w h.t $(=s n)$ jr $n=j w 3 . t$

jnk $\mathrm{Hr} \mathrm{3}^{\mathrm{c}}-\mathrm{hrw}$

$j y . n=j(r) n \underline{d}(. n=j) j t=j W \operatorname{sjr} W n-n f r\left(m 3^{c}-h r w\right) s 3 G b(m s . n)$ Nw.t

$(d j=j) w n(n) n \underline{t r} . w h \underline{h r} \underline{h n m}=f / \underline{h n m} m$

$m 33$ (sw) ntr.w (m) ch.t

$d j=s n n=f j 3 w(h r-t p t 3)$

$\mathbf{R} \quad w^{c} b z p \operatorname{sn.nw} W \operatorname{sjr}(N$.) (r) $z p f d w$

$j^{c j}(. n=f) h r=f m h z . t$ n.t $R^{c}$

hrw pwj $n$ wp.t-rnp.t

$j w j r . n=f 3 h$ jqr $m-b 3 h$ W Wjr

$m \underline{h b}=f n f r n \underline{h n m}$ h. $\underline{d} . t$

Die Strophen B-G sind in gleicher Form gestaltet, indem ihre vier Verse jeweils mit den gleichen Worten beginnen:

jy. $n=j \operatorname{mjn} h r s b 3 n \ldots$

$\mathrm{XY}, j r n=j w 3 . t$

jnk $\mathrm{Hr} \ldots$

$j y . n=j \ldots$

Ab Strophe $\mathrm{H}$ verändert sich der Text: bei $\mathrm{H}$ fehlt zunächst nur die letzte Zeile $j y . n=j$ ..., während in den folgenden Abschnitten I bis O der Text bis auf zwei Zeilen reduziert wird. Eine vergleichbare Verkürzung der Texte zum Ende hin ist übrigens in der Kurz-

39 Die Quellen sind an dieser Stelle sehr schwer lesbar und unverständlich.

$40 \mathrm{Im}$ Text des 10. Tores von Tb 146 begegnet ein shn wr. 
fassung des Amduats zu beobachten ${ }^{41}$. Die Abschnitte I und K bilden einen Sonderfall, da die Möglichkeit besteht, daß sie ursprünglich auf folgende Weise zusammengehörten:

$j y . n=j$ mjn $h r$ sb3 $n \underline{t} \underline{p h . t}$

$r h . k w j$ sšt $3 . w n t j j m=s t j y . n=j m j n c_{3} / d j m$ njw.t wr.t

jnk $R^{c} m d w 3$

$d j=j \underline{t} 3 w n{ }^{c} n \underline{h} n(j t=j) W_{s j r}$

Die abweichende Gestaltung dieser Strophe hat anscheinend bereits den ägyptischen Bearbeitern Schwierigkeiten bereitet. Ab der Quelle Irti. erscheint der Abschnitt I hinter E, so daß K der unvollständigen Strophe $\mathrm{H}$ folgt. Aufgrund dieser Trennbarkeit der Abschnitte $\mathrm{I}$ und $\mathrm{K}$ wurden für die vorliegende Bearbeitung zwei verschiedene Großbuchstaben vergeben, obwohl zu bedenken bleibt, daß $\mathrm{K}$ somit der einzige Abschnitt zwischen B und Q ist, der nicht die Nennung des Tores $(s b 3)$ in der ersten Zeile enthält und daher vielleicht ursprünglich keine eigene Strophe bildete. Die Abschnitte LMNO sind danach nur zweizeilig, wobei die zweite Aussage bei L wie bei I gestaltet ist $(r h . k w j \ldots)$, während die anderen drei in der Anrufung von Göttern bzw. Dämonen und der folgenden Aufforderung $j r n=j w 3 t$ miteinander parallel sind und dem Beginn der «Standardstrophe» entsprechen.

Es bleiben als letzte Tor-Strophen die Abschnitte P und Q. Sie sind in den Quellen R und $\mathrm{T}$ am ausführlichsten gestaltet, folgen dort aber wie in Irti., Iah. und $\mathrm{L}$ nicht aufeinander, wie es in den vier ersten Quellen belegt ist, sondern Q steht hier zwischen C und $\mathrm{D}$ (siehe dazu unten). Während $\mathrm{P}$ in seiner formalen Gestaltung am wenigsten den sonstigen Abschnitten gleicht, liegt in $\mathrm{Q}$ zunächst wieder die vierzeilige Standardstrophe vor. Anschließend enthält sie drei Verse, die die Wirkung des Spruches auf die Götter beschreiben, so daß Abschnitt Q mit diesen zusammenfassenden Aussagen tatsächlich an den Schluß aller Tore zu gehören scheint, wie es in den vier ersten Quellen richtig bezeugt ist.

Die Reihenfolge der Abschnitte ist in den Quellen also folgendermaßen überliefert (die Sternchen bezeichnen Kurzformen der Abschnitte, siehe dazu im Einzelnen unten:

\begin{tabular}{|c|c|c|c|c|c|c|}
\hline $\mathrm{Cg}$ & $A$ B C & $\mathrm{E}$ & F G H I & $\mathrm{KL} \mathrm{MNO}$ & $\mathrm{P}^{*}$ & $2^{*}-$ \\
\hline Ec & $A$ B C & D E & F G H I & K L MNO & $\mathrm{p} *$ & * $R$ \\
\hline Pef. & $A$ B C & D E & F G H I & K L MNO & $\mathrm{P}^{*}$ & $\mathrm{Q}^{*}$ \\
\hline Nes. & $A$ B C & D E & F G H I & K L MNO & $\mathrm{P}^{*}$ & $\mathrm{Q}^{*}$ \\
\hline Irti. & $A$ & E I & $\mathrm{F} / / /$ & $1 / 1 / 1$ & & 11 \\
\hline h. & $A$ & Q D E I & $\mathrm{F}$ & K L MNO G* & $\mathrm{P}^{*}$ & \\
\hline $\mathbf{R}$ & $A$ & E I & F G H & K L MNO & $P$ & \\
\hline 1 & $A \mathrm{~B} \mathrm{C} \mathrm{Q}$ & Q D E I & F G H & $\mathrm{K} \mathrm{L} \mathrm{MNO}$ & $\mathrm{P}$ & \\
\hline & $A B C O$ & Q D E I & F $\quad \mathrm{H}$ & K L MNOG & $\mathrm{P}$ & \\
\hline
\end{tabular}

41 Vgl. dazu Derchain, Rez. zu Hornung, Das Amduat, III, (̈̈g. Abh. 13, 1937), in: BiOr 25 (1968), 321. 
Es zeigt sich, daß Einleitung $(A)$ und $\operatorname{Schlu} \beta(R)$ in ihrer Position konstant bleiben und daß die Strophengruppen BC, DE und KLMNO fester zusammengehörten bzw. einheitlicher überliefert wurden als die übrigen Strophen, die an verschiedenen Stellen auftauchen können. Neben dem Vorziehen der kurzen Strophe I ist es vor allem die Position der langen Sequenz Q, die durch ihre wahlweise Position die acht Quellen in zwei Überlieferungsgruppen teilt: eine ältere, die sie vor die Schlußstrophe setzt, vielleicht als Zusammenfassung der vorherigen Aussagen; eine jüngere, die sie am Anfang hinter C plaziert, wo sie aufgrund ihrer Struktur ebenfalls gut aufgehoben erscheint. Es ist jedoch zu bemerken, daß die ältere Gruppe die Strophe Q nicht vollständig tradiert. Wenn die Rekonstruktion richtig sein sollte, ist das Ende von $\mathrm{P}$ (hrj.w njw.t jr $n=j$ w3.t h̆m sšm $=j$ $t w=j)$ und der Beginn von Q (jy.n=j mjn hr sb3 $n$ hp.t wr) dort ausgelassen. Nimmt man die jüngeren, ausführlicheren Texte als Zeugen für den ursprünglichen Textbestand, so ist anzunehmen, daß die paläographische Ähnlichkeit einiger Wörter zur Auslassung bei einer frühen Vorlage geführt hat, von der nach und nach die ältere Gruppe abstammt: der letzte, allen Textzeugen gemeinsame Satz in P lautet

$$
j y . n=k \quad h r \text { shn jr.t } \mathrm{Hr} \text {, }
$$

die erste Zeile von Strophe Q (die in den älteren Quellen ausgelassen ist) heißt $j y . n=j \min$ hr sb3 $n$ hpt wr.

Abgesehen vom unterschiedlichen Suffix, dem Zeitbegriff mjn (der des öfteren ausgelassen oder an falschen Stellen eingesetzt worden ist) und dem Einschub $s b 3 n$ gleichen sich beide Sätze in der Bedeutung und Determinierung der Verben shn und hpt und in den Zeichen für die Vogelarten Schwalbe (wr) und Falke $(H r)$, so daß hier aberratio oculi zur Auslassung der Passage geführt haben könnte. Auch die Fortsetzung ist verwechselbar: in $\mathrm{P}$ sind es die $h r j . w n j w . t$ (bei Quelle L: hrj.w $\underline{h} . t=s n$ ), determiniert mit der Schlange, in Q die $h r j . w \underline{h} . t(=s n)$, determiniert mit Schlange bzw. Schakal, Schlange und Krokodil. Der älteren Quellengruppe läge also eine Vorlage zugrunde, die die betreffende Textpassage ausgelassen hat (bei $\mathrm{Cg}$ finden sich übrigens zwei $g m$-wš-Spatien in der Nähe), während die jüngere auf einer Vorlage basiert, die den Text zwar vollständig enthälte, die Strophe $\mathrm{Q}$ aber nach vorne gezogen hat.

Die beiden Tradierungsgruppen " $\mathrm{Cg}$ bis Nes.» und «Irti. bis L» werden durch weitere Varianten voneinander getrennt, von denen die Ersetzung der mittelägyptischen Vokabel c3 «hier» durch die neuägyptische $d j$ vielleicht die aussagekräftigste ist ${ }^{42}$ :

\footnotetext{
42 Die folgenden Übersichten richten sich in den Kennzeichnungen der Lesarten nach Schenkel, Das Stemma der altägyptischen Sonnenlitanei. (GOF IV/6, 1978), S. 52 f: 1 = Lesart 1, 2 = Lesart 2, 0 = zerstört, - = nicht enthalten, $\mathrm{S}=$ "spezielle» Individual-Lesart.
} 
$\mathrm{K}: \quad c_{3}(1), \operatorname{dj}(2)$

Q2: $\varnothing(1), m s n(2)$

$\mathrm{Q} 2: \varnothing(1), d j=j(2)$

Q2: Ø (1), $m(2)$
$\mathrm{Cg}$ Ec Pef. Nes. Irti. Iah. R T L

$\begin{array}{lllllllll}1 & 1 & 1 & 1 & 0 & 2 & 2 & 2 & \mathrm{~S}\end{array}$

$\begin{array}{lllllllll}1 & 1 & 0 & 0 & 2 & 2 & 2 & 2 & 2\end{array}$

$\begin{array}{lllllllll}1 & 1 & 1 & 1 & 0 & 2 & 2 & 2 & 2\end{array}$

$\begin{array}{lllllllll}1 & 1 & 1 & 1 & 0 & 2 & 2 & 2 & 2\end{array}$

In Bezug auf die Behandlung des zusammengesetzten Ausdrucks $n \underline{d} j t=f$, der in der Spätzeit immer häufiger mit der Präposition $h r$ gebildet wird, kann man in Ec, Pef. (?), Nes. (?) und Iah. vielleicht eine Tendenz zur Gewohnheit des Schreibers erkennen, die übrigen Quellen lassen keine Interpretation zu:

$\mathrm{Cg}$ Ec Pef. Nes. Irti. Iah. R T L

$\begin{array}{lllllllllll}n \underline{d} j t=f(1), n \underline{d} h r j t=f(2) & \mathrm{B}: & 1 & 2 & 2 & 0 & 2 & 1 & 2 & 2 & 2 \\ & \text { C: } & 2 & 2 & 0 & 1 & 1 & 1 & 2 & 2 & 2 \\ & \text { D1: } & 2 & 2 & 2 & 1 & 0 & 1 & 1 & 2 & 2 \\ & \text { Q1: } & 2 & 2 & 2 & 1 & 0 & 1 & 1 & 1 & 1\end{array}$

Die Quelle Iah. zeigt zusammen mit L in mehreren Punkten eine gesonderte Überlieferung: in beiden Textzeugen ist die Strophe G hinter O plaziert (vielleicht auch bei Irti., der Text ist an entsprechender Stelle leider zerstört). Außerdem wird anstelle von $j y$ «kommen» bei Iah. immer, bei L bis auf zwei Ausnahmen ${ }^{43} j w$ "kommen» geschrieben. Schließlich gibt es die beiden Leitvarianten:

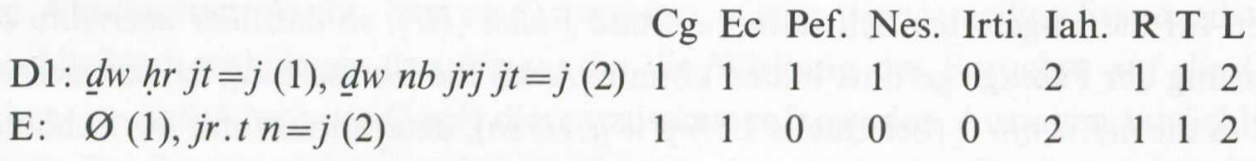

Die Quellen Iah. und/oder L gehen an manchen Stellen auffällig eng mit dem älteren Textzeugen $\mathrm{Cg}$ zusammen. Zu Beginn des Abschnitts D findet zich z.B. die Formulierung $h r s b 3 n$ d3.t $n b^{44}$ bei $\mathrm{Cg}$ und Iah., und bei L liest man: $h r s b 3 n$ d3.t nb.w d3.t. Alle anderen Versionen erwähnen an dieser Stelle zusätzlich oder stattdessen das «Tor des Westens» ( $s b 3 n j m n . t t)$. In $\mathrm{F}$ ist nur bei $\mathrm{Cg}$ und Iah. $j w^{c} n$ zwischen $W s j r$ und $W n-n f r$ eingeschoben. Weitere Belege für die Zusammengehörigkeit von $\mathrm{Cg}$, Iah. und $\mathrm{L}$ sind folgende Lesarten:

$\mathrm{K}: j t=j W \operatorname{sjr}(1), W s j r(2)$
$\mathrm{M}: s r . w(1), h n^{c} s r . w(2)$

$\mathrm{Cg}$ Ec Pef. Nes. Irti. Iah. R T L

$$
\text { M: } s r . w(1), h n^{c} s r . w(2)
$$

$\begin{array}{lllllllll}1 & 2 & 0 & 0 & 0 & 1 & 2 & 2 & 1 \\ 1 & 2 & 0 & 1 & 0 & 1 & 2 & 2 & 1\end{array}$
$379 \mathrm{ff}$

${ }^{44}$ Die Substantive stehen bei Iah. allerdings alle im Plural. 
Aus diesen und den oben aufgelisteten Beispielen wird außerdem deutlich, daß Ec, R und $\mathrm{T}$ zu einer Traditionslinie gehören. Die Zuordnung von Pef., Nes. und Irti. fällt in diesen Punkten schwer, da zu oft entscheidende Passagen zerstört sind.

Interessant ist des weiteren, daß $\mathrm{R}$ und $\mathrm{T}$ an einer Stelle eine mit dem Ausdruck $k y-\underline{d} d$ verknüpfte Kombination zweier Lesarten hat, die in älteren Texten einzeln belegt sind:

\begin{tabular}{|c|c|}
\hline P: $\mathrm{Cg}, \mathrm{Ec}, \mathrm{Nes}$ : & $s g 3 \check{s} y . t$ \\
\hline Pef., Irti.: & $\varnothing$ \\
\hline Iah.: & sgry.t \\
\hline $\mathrm{R}$ : & sg3šy.t ky $d d$ sqryw.t \\
\hline $\mathrm{T}$ : & $s g 3 \check{s} y . t k y \underline{d} d$ jqryw.t \\
\hline L: & jqry.t \\
\hline
\end{tabular}

Die Neigung zur Aufnahme von $k y-\underline{d} d$-Varianten (vgl. auch in Abschnitt $\mathrm{N}$ h.t $k y \underline{d} d d$ $s \underline{d} . t$ bei Quelle R) bezieht sich einmal sogar nur auf das Determinativ:

$\mathrm{L}$ : Cg, Ec, Pef.: qmdy.t mit Det. «schlechtes Paket» bzw. «Ei»

Nes.: $\quad q 3 . w t$

Irti.: $\quad \varnothing$

Iah.: $\quad q 3 y . t$ mit Det. «Käfer»

$\mathrm{R}, \mathrm{T}$ : $\quad q 3 w y . t$ mit Det. «Sonnenscheibe» $k y \underline{d} d$ Det. «Skorpion»

$\mathrm{L}: \quad q 3 y . t$ mit Det. «Skorpion»

In Bezug auf singuläre Lesarten fällt besonders die Quelle Iah. ins Auge: in B schreibt sie als einzige $j w . n=j h \boldsymbol{n}^{c} \boldsymbol{h}^{c} \cdot \boldsymbol{w t} h r \quad W s j r^{45}$, in Strophe G wird nur der erste Satz geschrieben, der Rest ist ausgelassen. In N findet sich eine Variante mit Geb, am Ende vor $P$ entspricht der Textbestand weder der älteren Gruppe noch der jüngeren, sondern ist eine Mischung aus beiden. In Abschnitt $\mathrm{R}$ schließlich ist der Eigenname der Besitzerin ausgelassen, bei $j c j . n=f h r=f$ steht als Objekt $j b=f$ und der Text bricht nach $m h b=f$ ab. Diese Sonderstellung der Quelle Iah. läßt sich möglicherweise dadurch erklären, daß dieses Totenbuch aus der Gegend von Herakleopolis magna stammt ${ }^{46}$, während die anderen Quellen im thebanischen Raum angefertigt wurden. Da der überwiegende Textbestand dieses Zeugen allerdings eine große Nähe, besonders zu Ec und L, aufweist (siehe oben), ist die Entwicklung der mittelägyptischen Tradition auch nicht allzu selbständig von der thebanischen zu denken.

Als kurze inhaltliche Charakterisierung des Schlußtextes von Tb 146 sei abschließend angeführt, daß er zwar in thematischem Zusammenhang mit den benachbarten Tor- und Pforten-Kapiteln 145-147 steht, wie einige, oben zitierte Parallelen zeigen. Die Nachschrift (Abschnitt R), die die Reinigung des Königs aus der hz.t-Flasche des Re erwähnt, ist

\footnotetext{
${ }^{45} \mathrm{Cg}$ und Ec $\underline{d} h^{c}(h r)$, Pef. und Irti. zerst., Nes.: $<\underline{d}>w . t$ (?), R und T: $\underline{d n} h(\underline{d}) n h=k, \mathrm{~L}: d n d n n b$.
}

46 Vgl. Verhoeven, Das saitische Totenbuch, S. 5 f. 12. 
allerdings von Graefe in den religiösen Komplex «Erstes Bad, Krönung und Epiphanie des Sonnengottes» gestellt worden ${ }^{47}$. Ergänzend zu seinen überzeugenden Argumenten sei hier erwähnt, daß weitere Elemente dieses Themenbereichs im Hauptteil des Schlußtextes von $\mathrm{Tb} 146$ enthalten sind:

Die Tore, die hier übrigens $s b 3$ genannt werden und nicht $s b h . t$ wie die im Haupttext von $\mathrm{Tb}$ 146, entsprechen möglicherweise denen des Tempels und des Himmels (vgl. Graefe, 62); zur Reinigung mit $c_{n h}$ und w3s (Graefe, 64) vgl. hier Abschnitt E; das Aufsetzen der wrr.t-Krone (Graefe, 66) wird in Abschnitt A angesprochen; Horus, der den Dienst des «geliebten Sohnes» ausübt (Graefe, 67), begegnet in Abschnitt H, zum Bringen des Lichtes in der Nacht (vor dem Jahresanfang, Graefe, 71) siehe Abschnitt F.

Der Verstorbene, der diesen Spruch rezitieren soll, identifiziert sich in den diversen Strophen an den 15 Toren zumeist mit Horus, dem Sohn, Schützer und Erben seines Vaters Osiris. An zwei Toren findet jedoch eine direkte Gleichsetzung mit dem Sonnengott statt: in Abschnitt $\mathrm{G}$ heißt es jnk nhh $m$ h3.t wj3 ( $n R^{c}$ ) "Ich bin der Greis ${ }^{48}$ am Bug der Barke (des Re)» und in $\mathrm{K} j n k R^{c} m d w 3$ «Ich bin Re am Morgen». Eine Verschmelzung von königlicher und solarer Herrschaft und Erneuerung ist somit das Thema dieses Spruches, der in der vielschichtigen Tradition spätzeitlicher Totenbücher seinen redaktionellen Niederschlag gefunden hat.

\section{Résumé/abstract}

Observations sur la transmission du Livre des Morts après le Nouvel Empire, fondées sur l'édition synoptique de neuf exemplaires du chapitre $146 \mathrm{w}$ appartenant aux XXI ${ }^{e}, \mathrm{XXV} / \mathrm{XXVI}{ }^{e}$ dynasties et à l'époque ptolémaïque. Ce texte se répartit en dix-sept paragraphes dont l'ordre peut varier. On reconnaît ainsi plusieurs traditions.

Quinze de ces paragraphes sont consacrés à la description de "portes» (sb3). Sept d'entre eux contiennent une invocation à Horus et deux au dieu-soleil à qui s'identifie le défunt.

\section{Nachtrag}

In der inzwischen erschienenen Publikation von TT 353 stößt man auf den einzigen und somit ältesten Textzeugen aus dem Neuen Reich: P.F. Dorman, The Tombs of Senenmut, (PMMA 24, 1991), 119-20, Tf. 66-9, Z. S 66-SW 4. Der Schlußtext von Tb 146 steht dort isoliert, d.h. ohne die vorherige Aufzählung der 15 Pforten, und ist wie folgt gegliedert (der abschließende Paragraph R fehlt bei Sen. wie in der nächst älteren Quelle $\mathrm{Cg}$ ):

Sen. A BC Q DE I F H KLMNO G P*

Abgesehen von einzelnen Textvarianten (vgl. dazu Dorman, 119f.), die Aufschluß über den Urtext geben können, bestärkt die Fassung im Grab des Senenmut die aufgestellte Vermutung, $\mathrm{da} ß$ die Quellengruppe $\mathrm{Cg}$, Ec, Pef. und Nes. auf eine andere Vorlage zurückgeht als die jüngere Gruppe Irti, Iah., R, T und L. Deren Vorläufer scheinen in der Tradition des Senenmut-Textes zu liegen. Die auffällige Reihenfolge der Abschnitte findet sich nur bei Sen., Iah. und L (im sehr unterschiedlich gestalteten Textteil P ist Sen. der Fassung von Iah. am ähnlichsten) und eine der Leitvarianten (D1: $\underline{d} w n b j r j j t=j$ ) ist ebenfalls nur bei diesen drei Textzeugen vorhanden.

47 Graefe, Der «Sonnenaufgang zwischen den Pylontürmen», in: OLP 14 (1983), 55-79.

48 Als Bezeichnung des in der Barke stehenden Sonnengottes am Abend: Hornung, Das Amduat, II, ( $\ddot{A g}$. Abh. 7, 1963), S. $21 \mathrm{f}$. 
TOTENBUCHSPRUCH 146

179

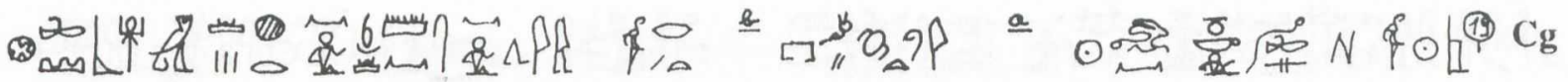

A

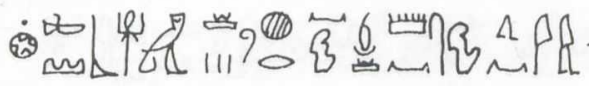

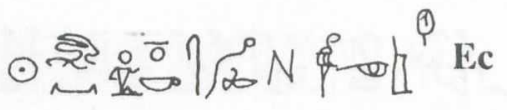

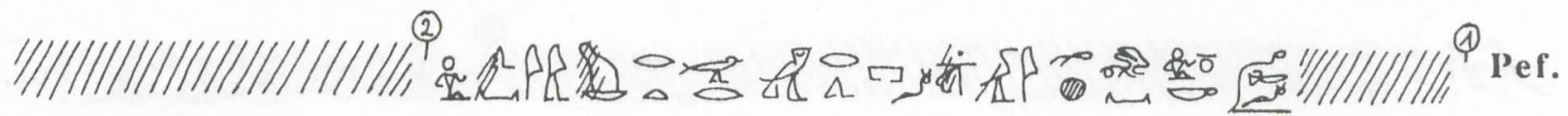

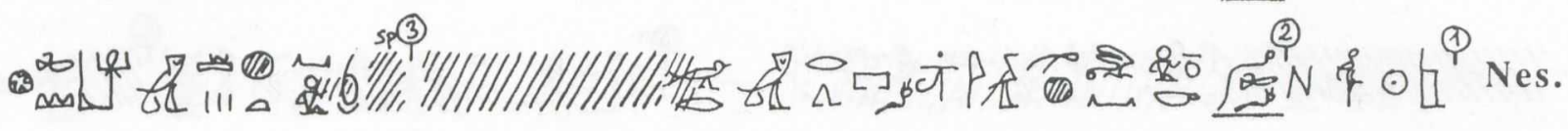

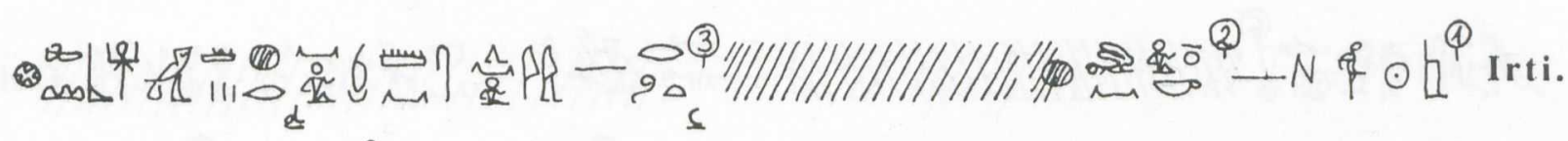

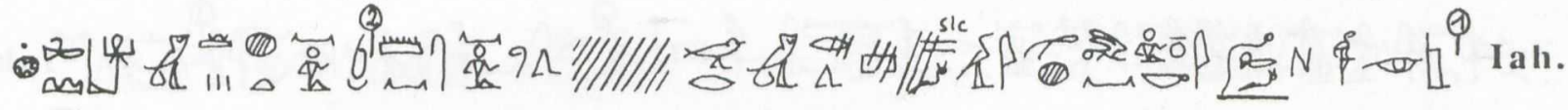

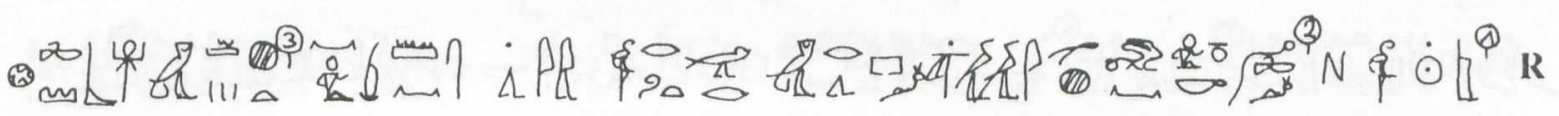

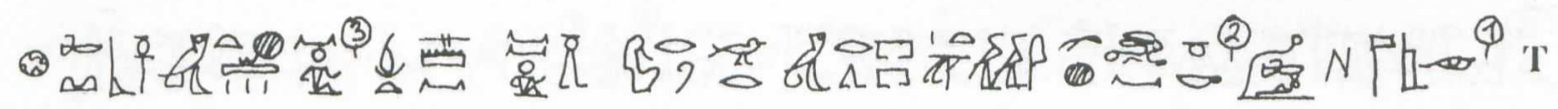

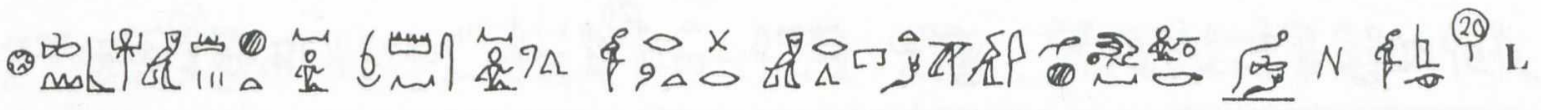

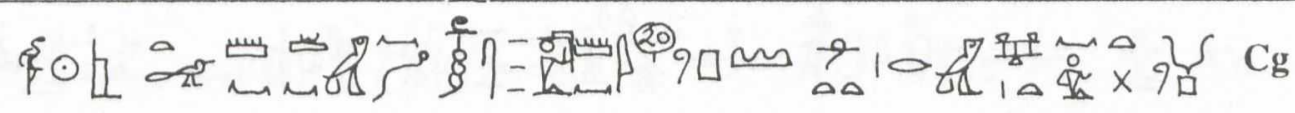

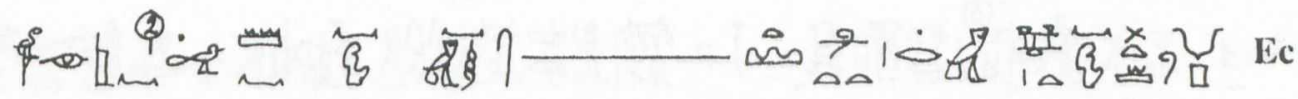

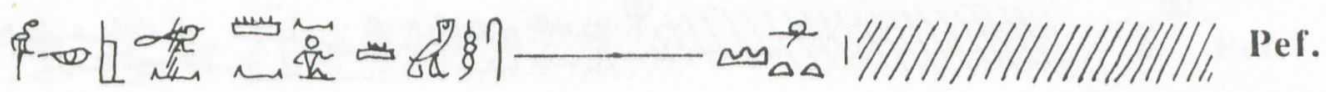

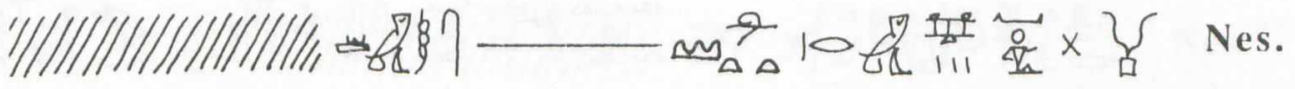

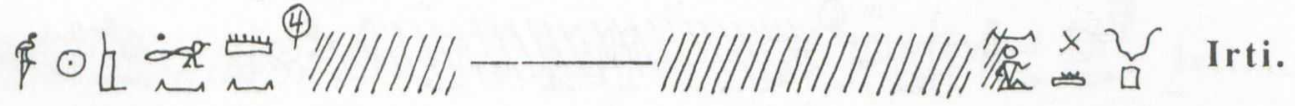

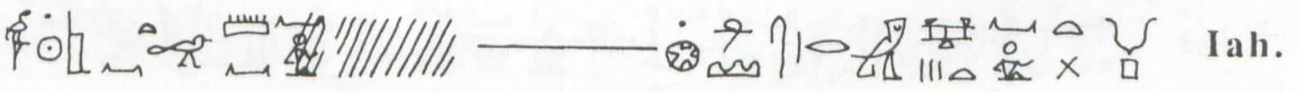

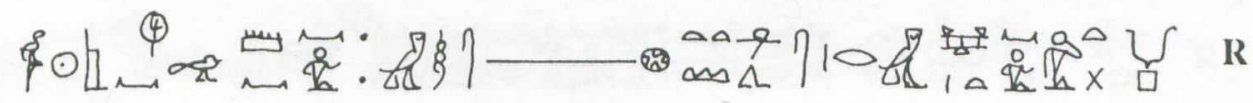

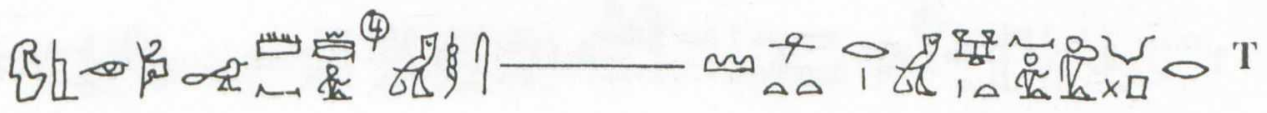

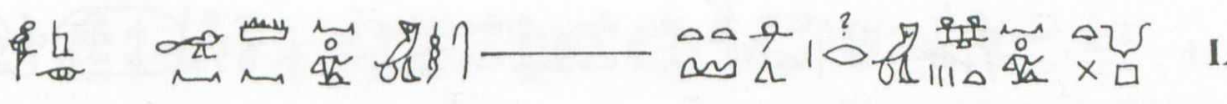

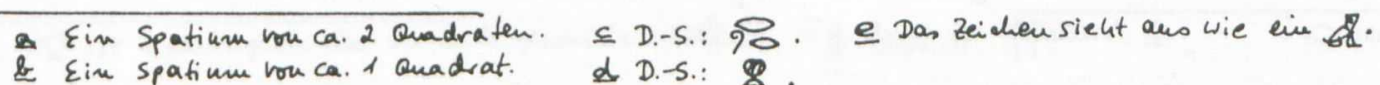




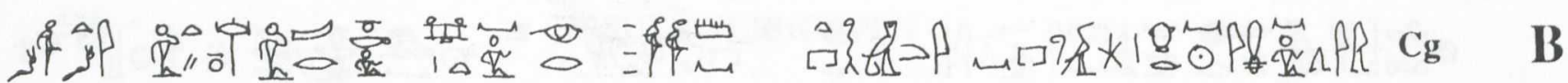

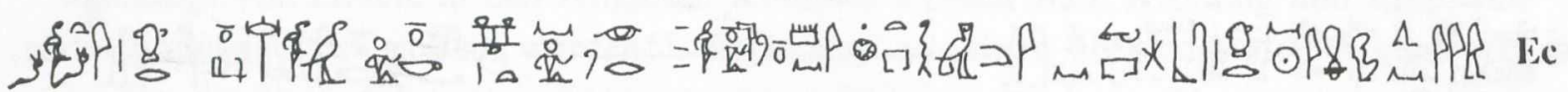

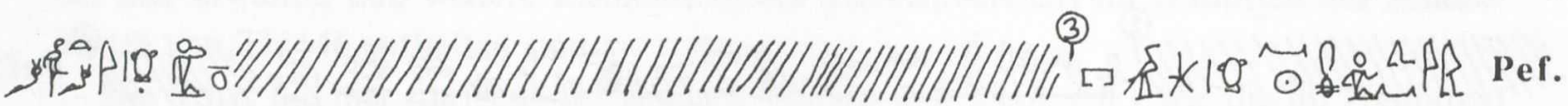

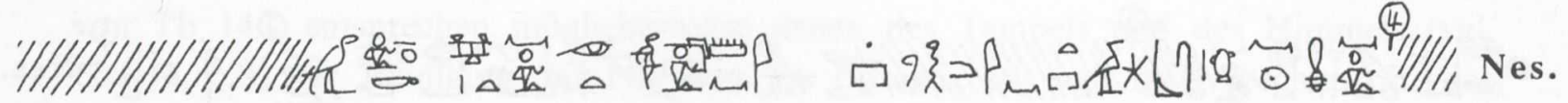

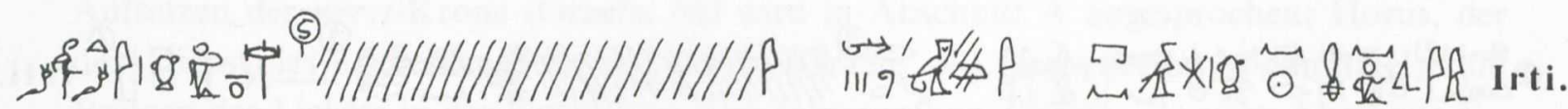

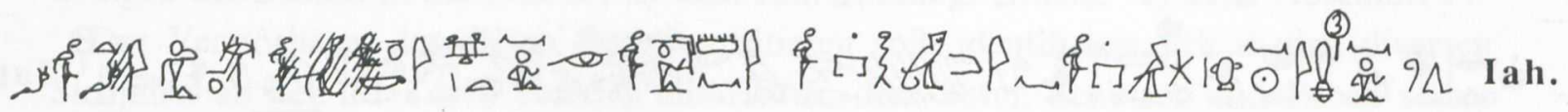

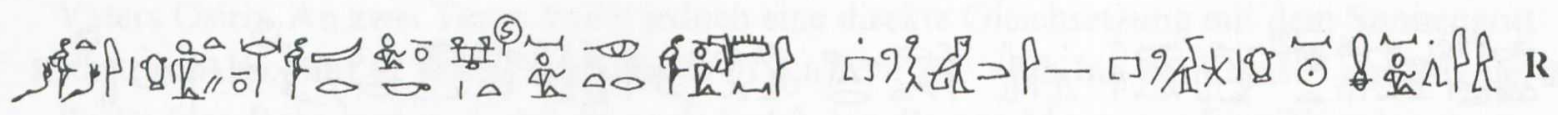

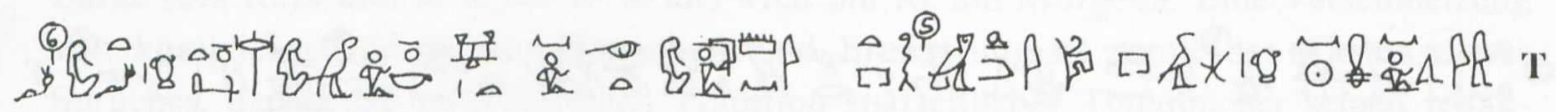

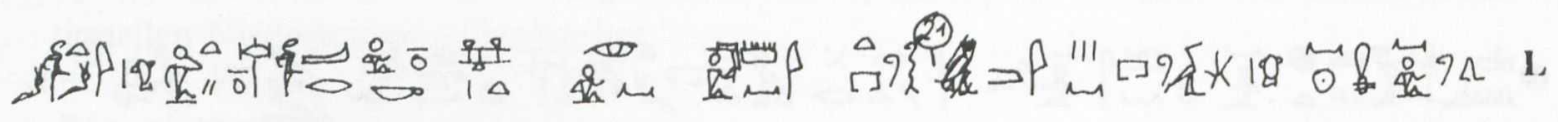

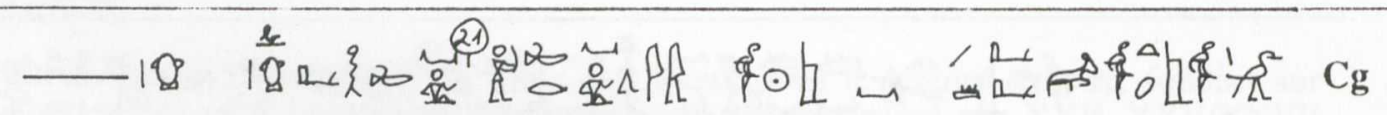
क्र०⿴囗十

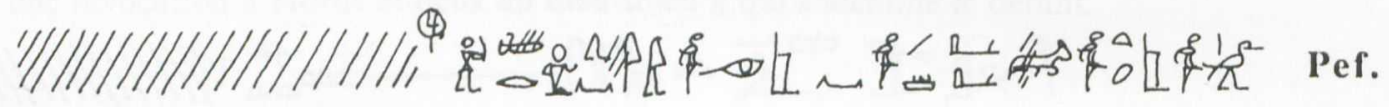

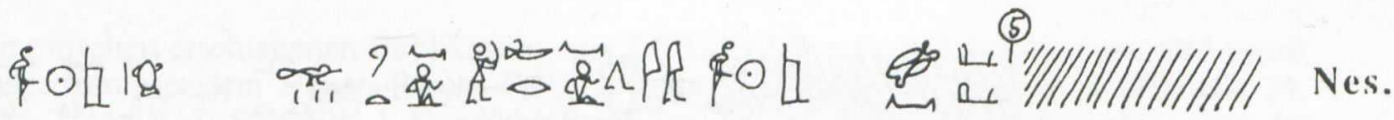

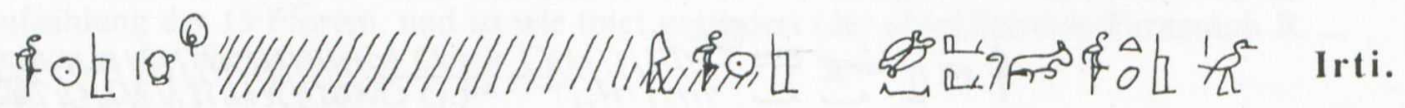

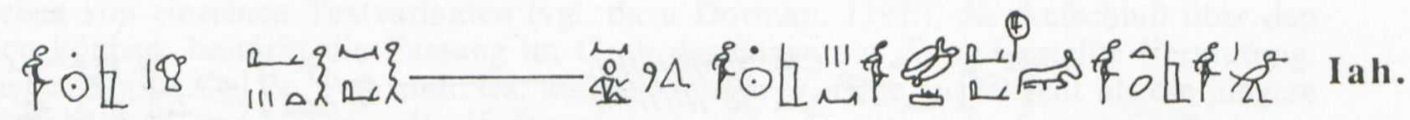

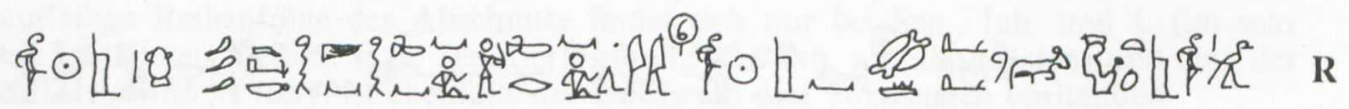

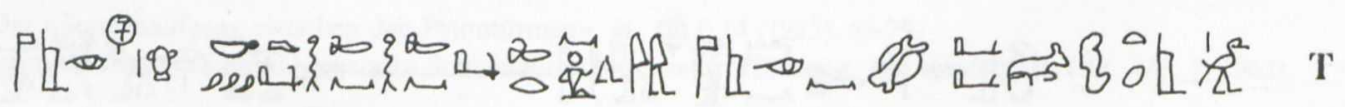

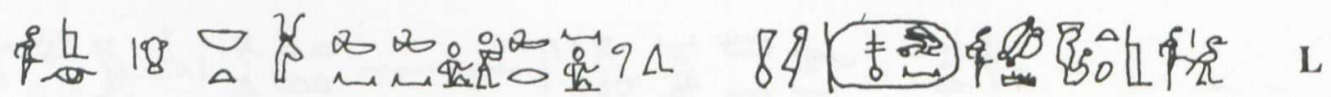


TOTENBUCHSPRUCH 146

181

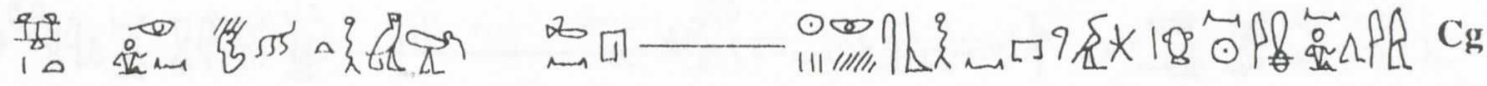

C

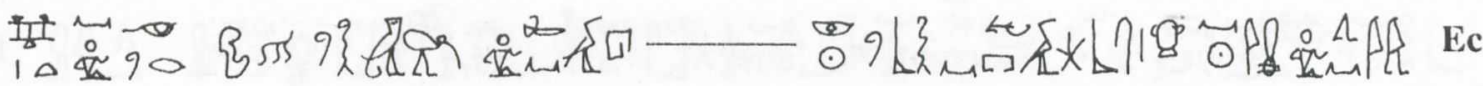

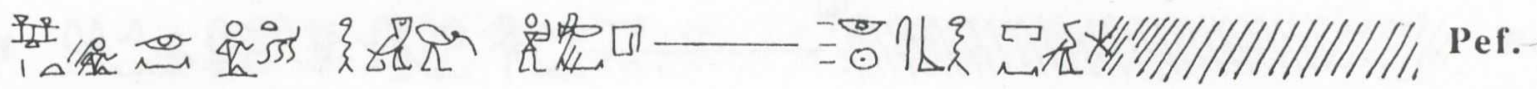

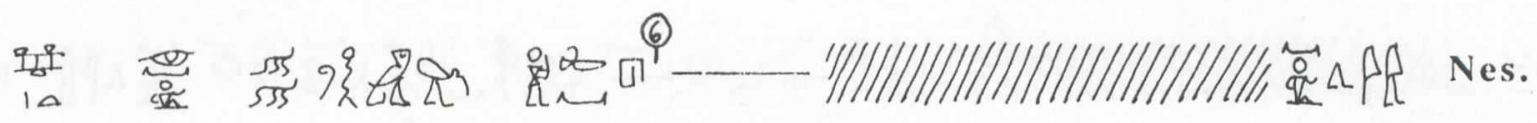

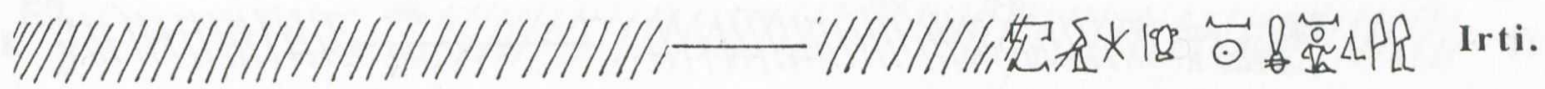

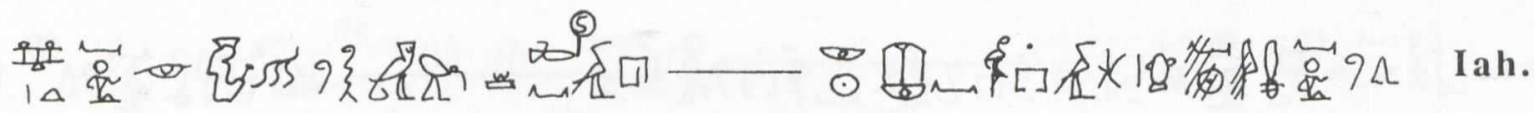

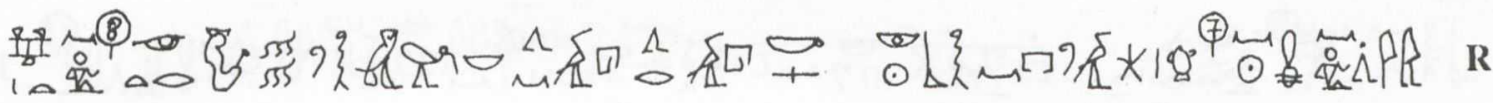

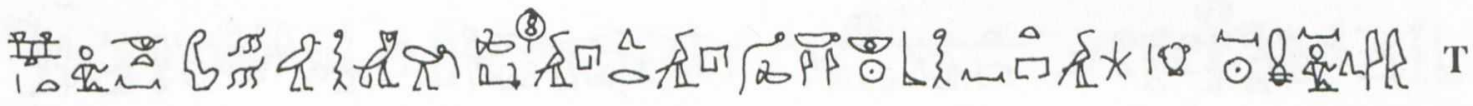

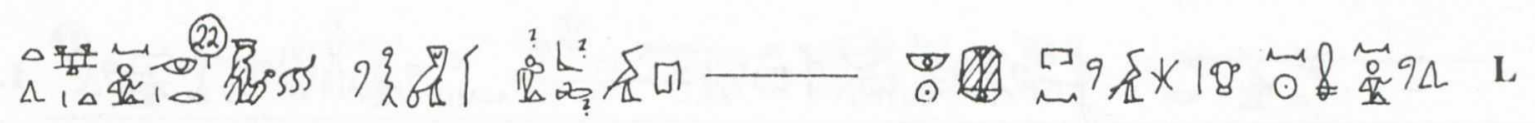

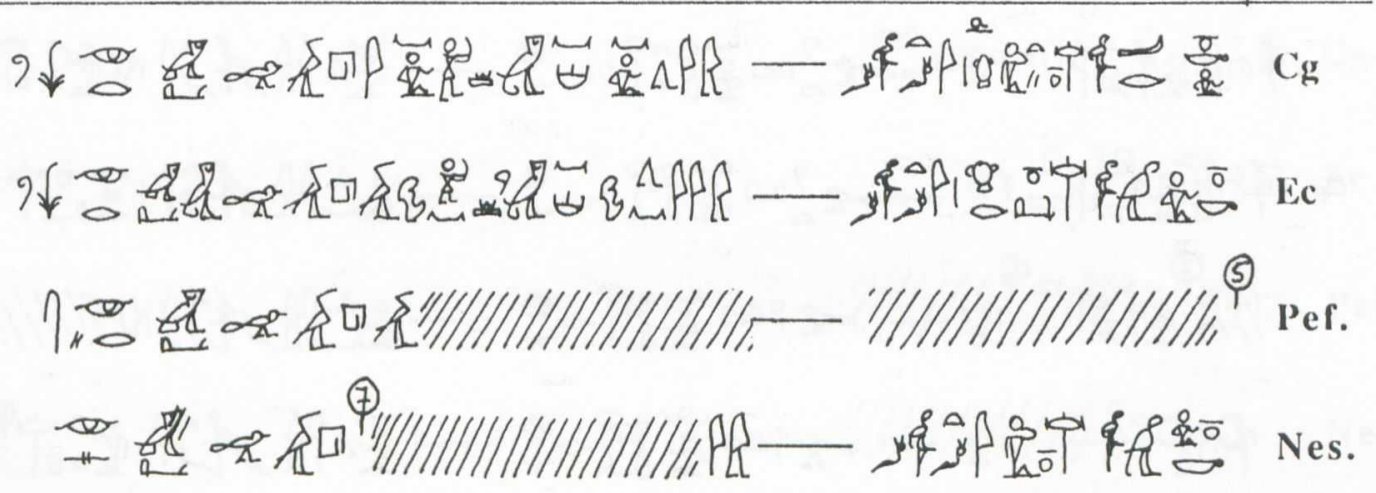

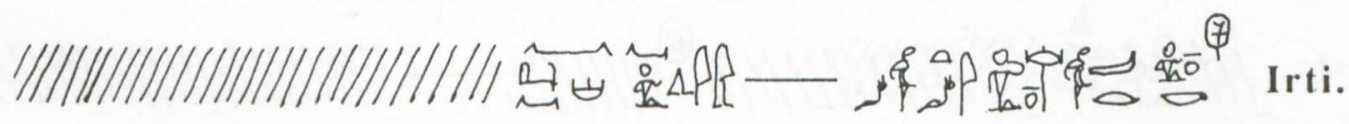

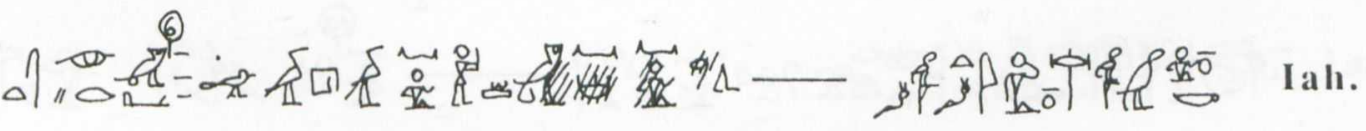

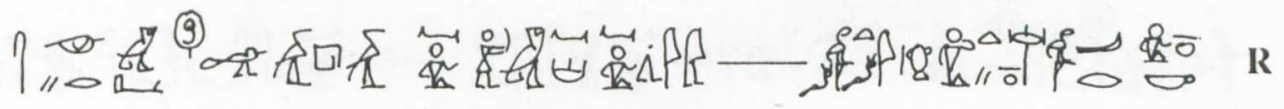

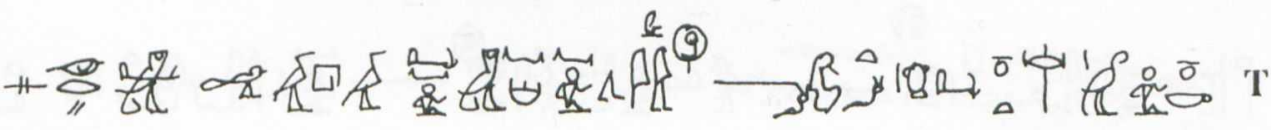

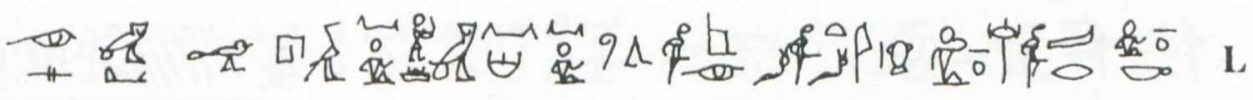

a 19 ist nachträglich wher die zeile opdrieten worden. \& Lepsius: AP. 
182

URSULA VERHOEVEN

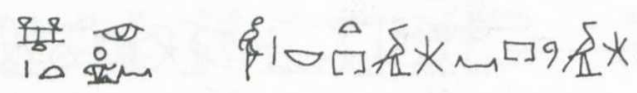

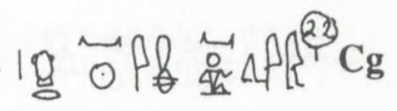

$\mathbf{D}_{1}$

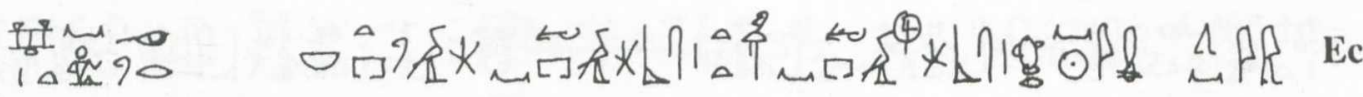

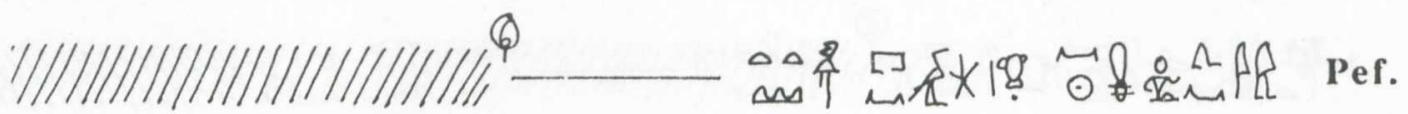
"IIII//II/I/I//禹?口秋

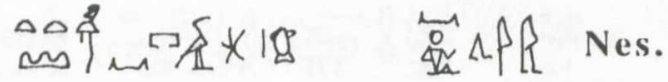
in $1 / / / / / / / / / / /\left[C^{(10)} \operatorname{Irti}\right.$.

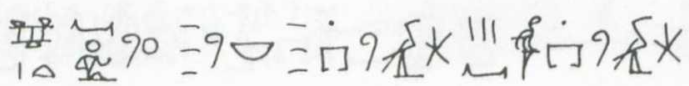

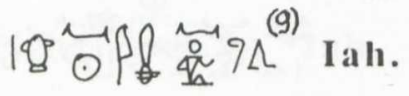

is

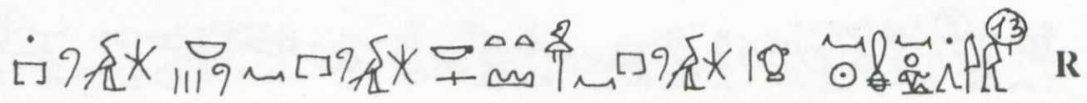

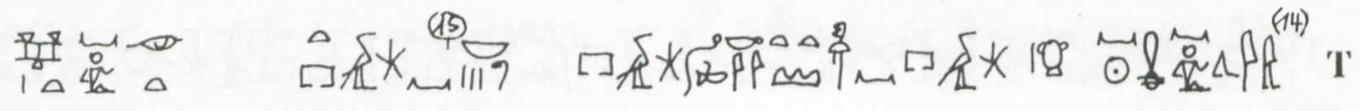

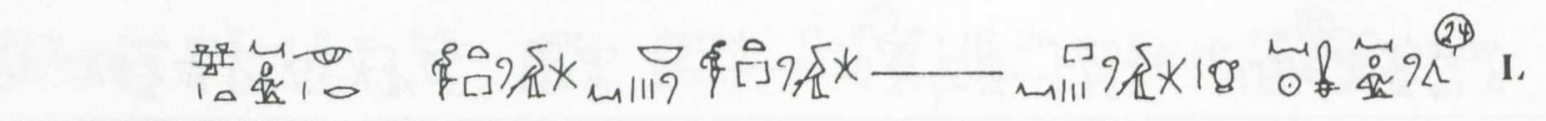

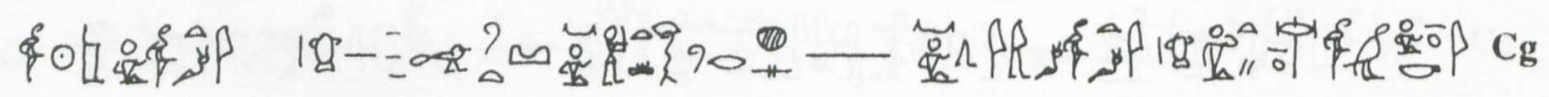

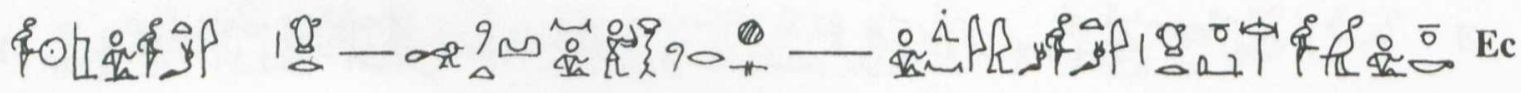
/li

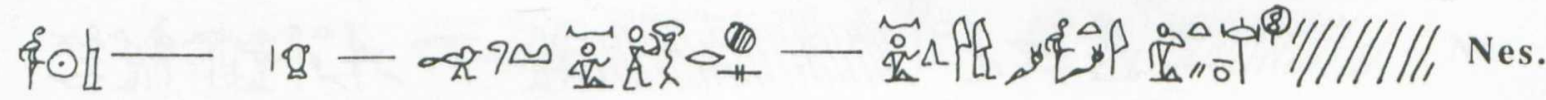

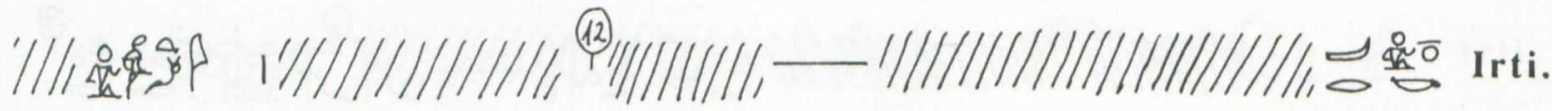

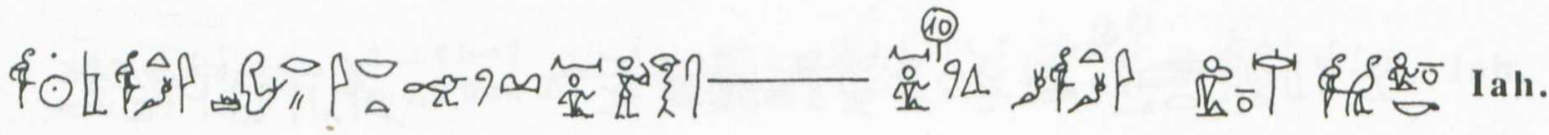

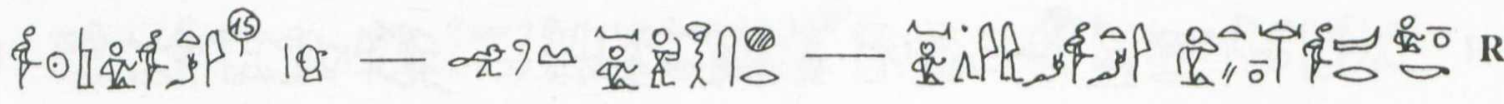

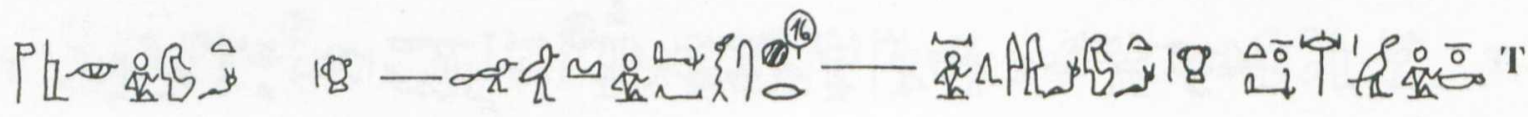

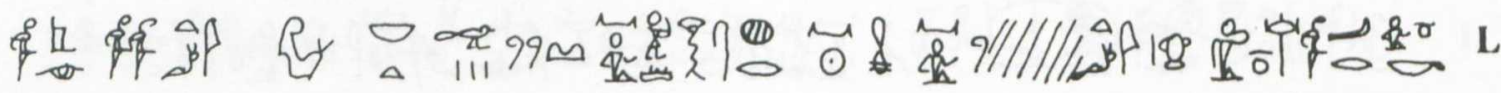




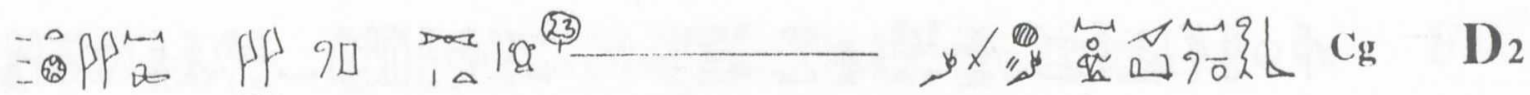

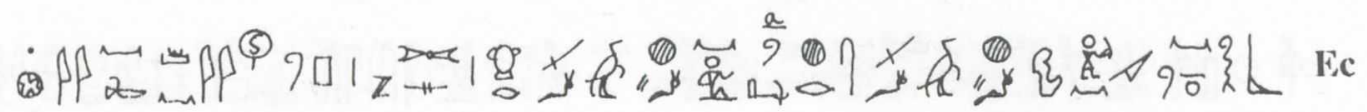

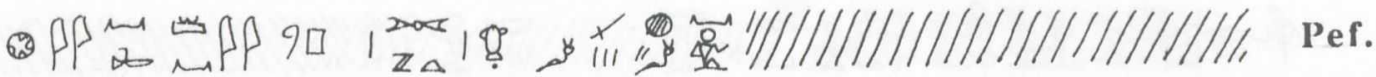

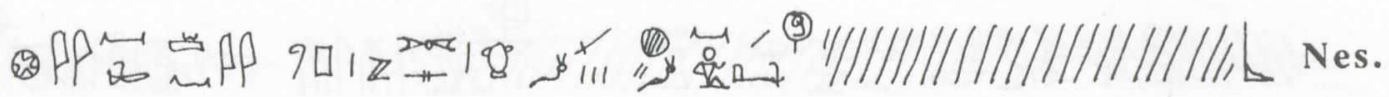

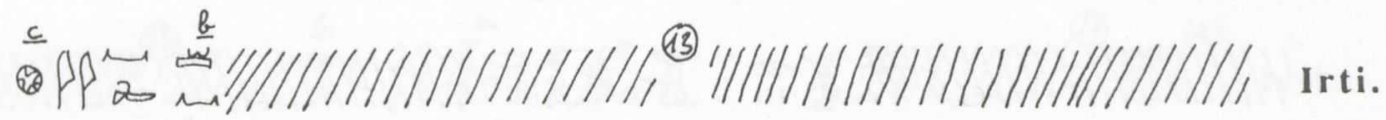

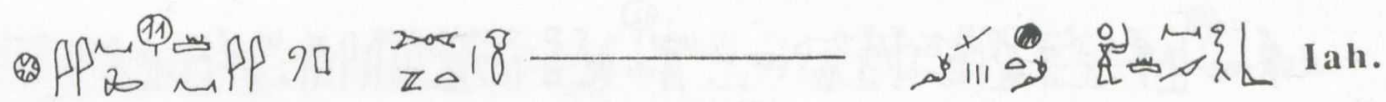

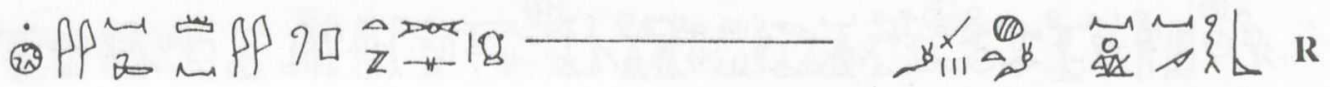
-

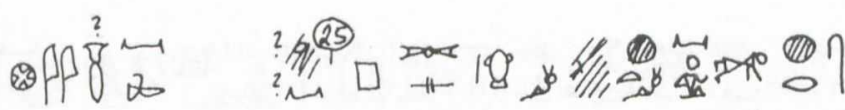

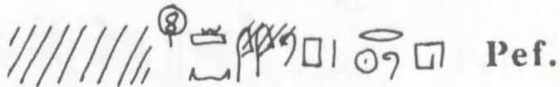

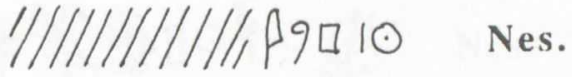

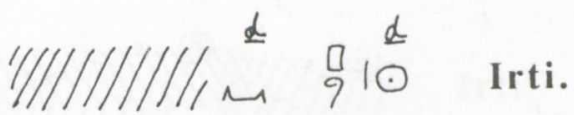

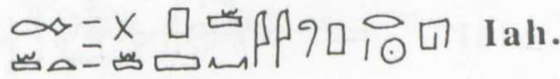

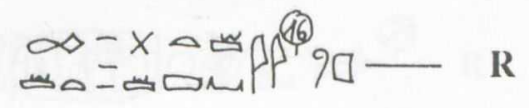

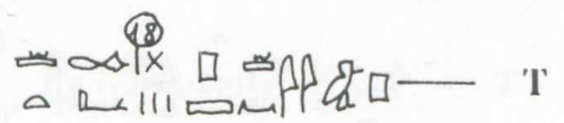

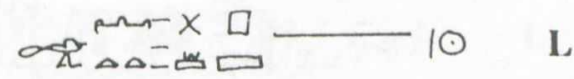

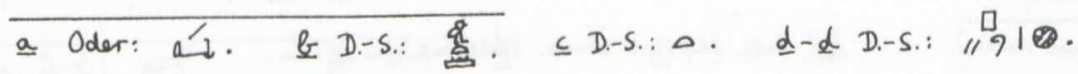




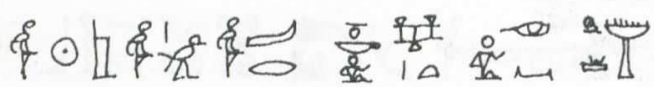

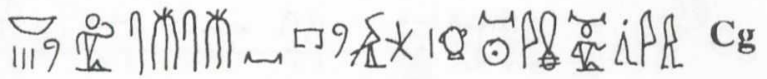

E

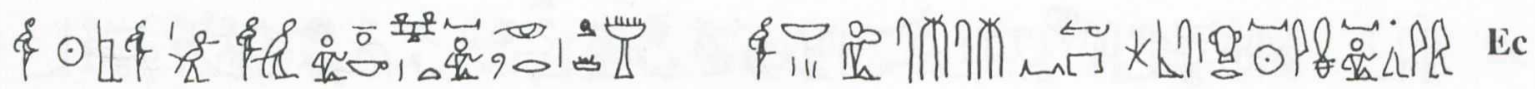

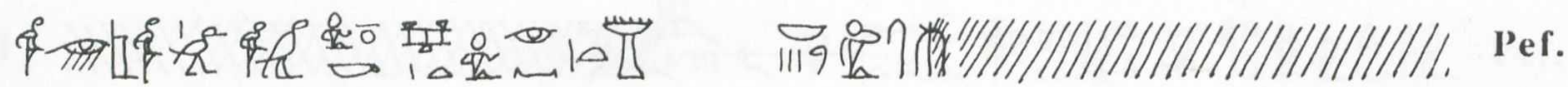

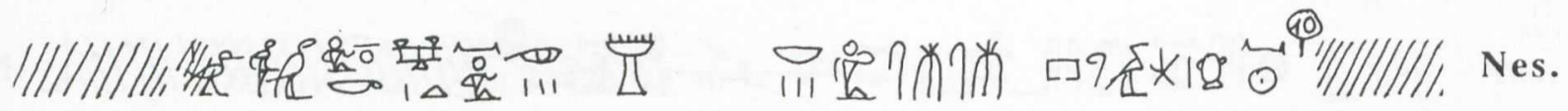

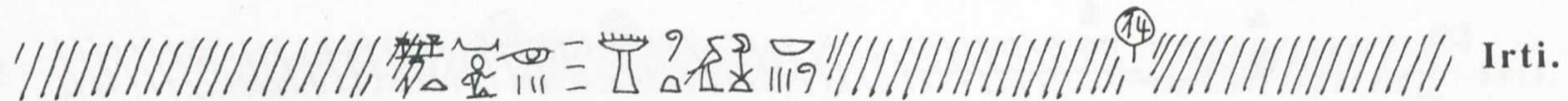

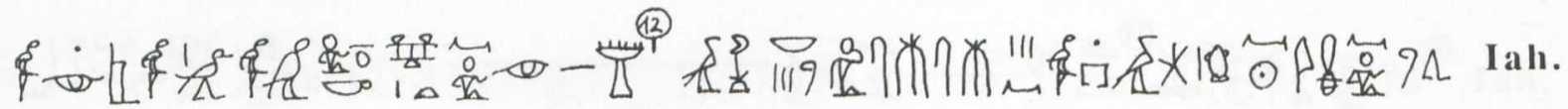

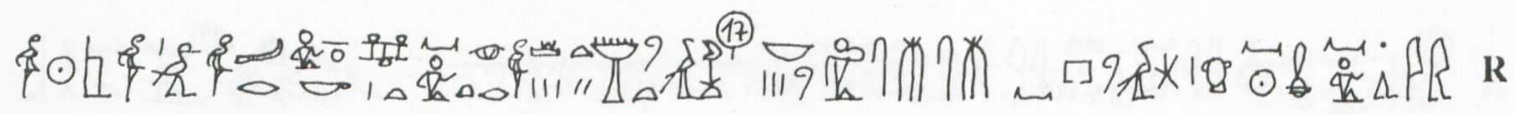

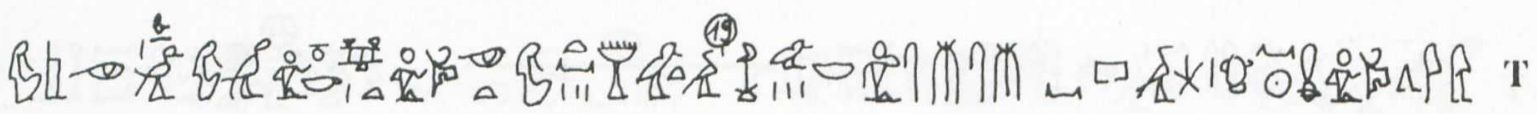

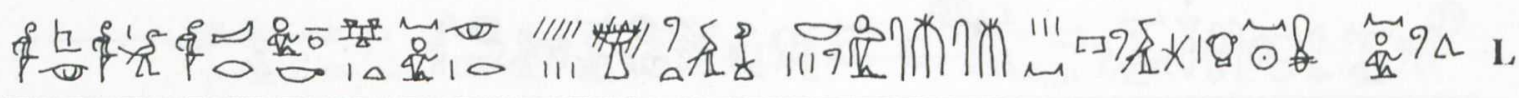

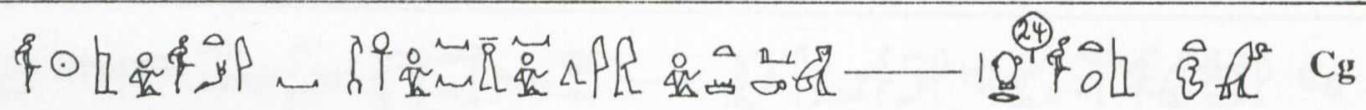

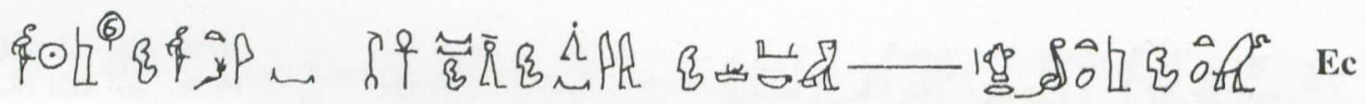

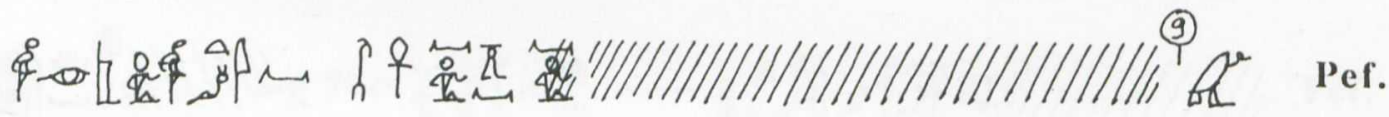

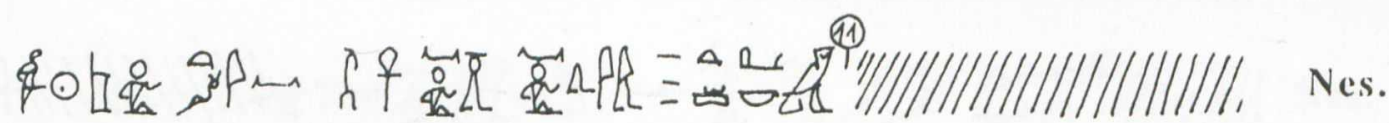

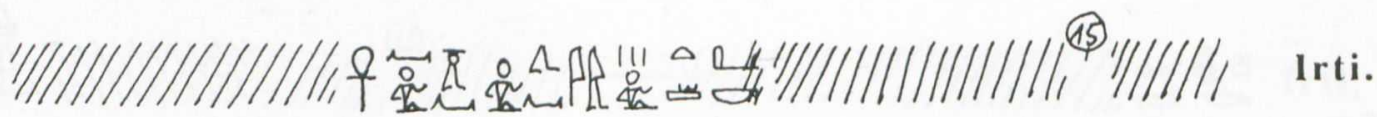

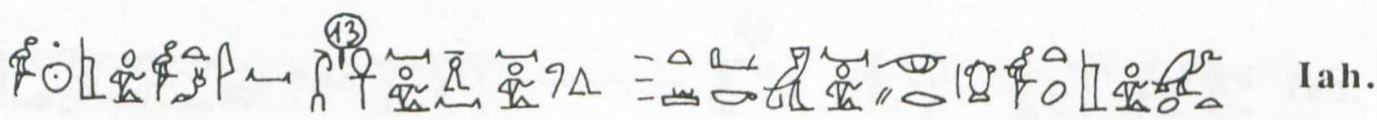

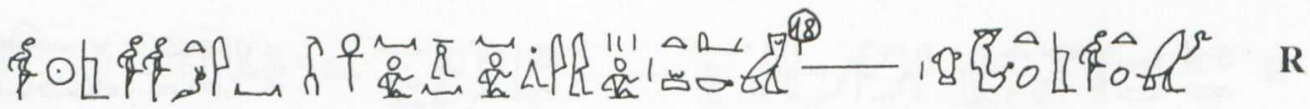

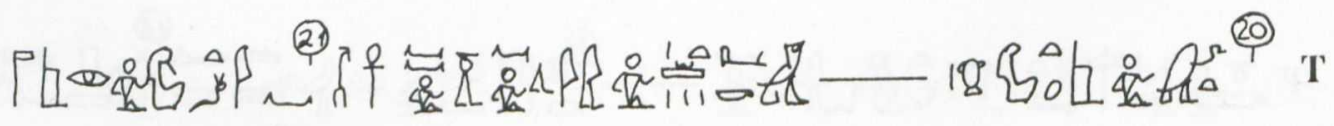

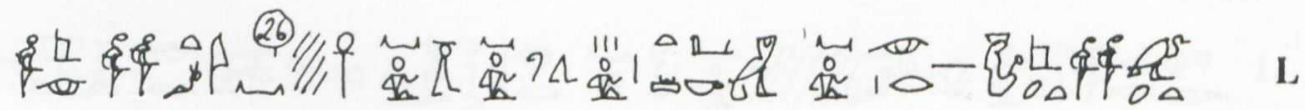

a Oder: $\mathbb{Z}$ (als Determinativ). If Bei lepsins olue Ideogramustrich. 


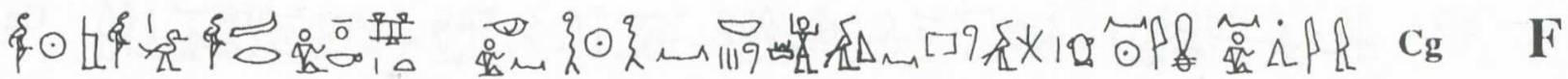

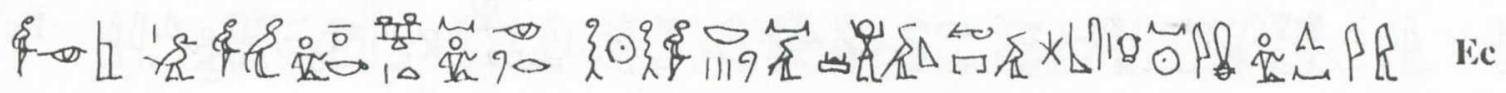

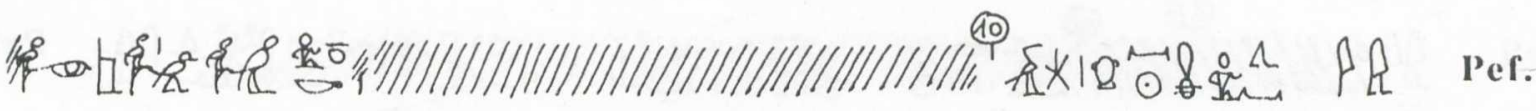

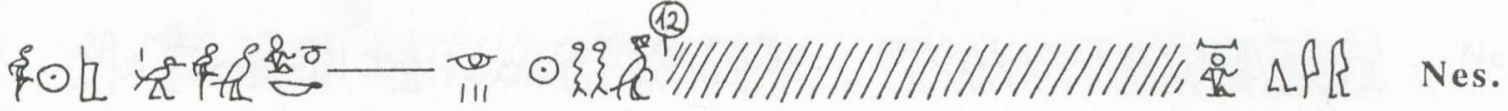

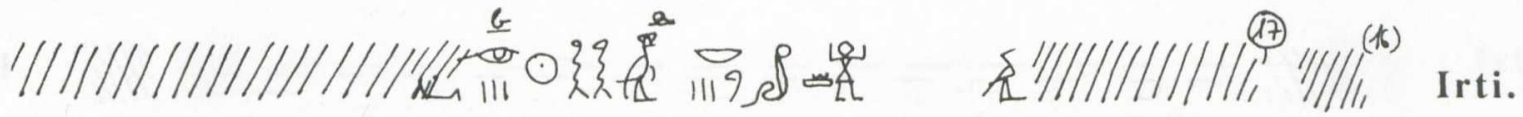

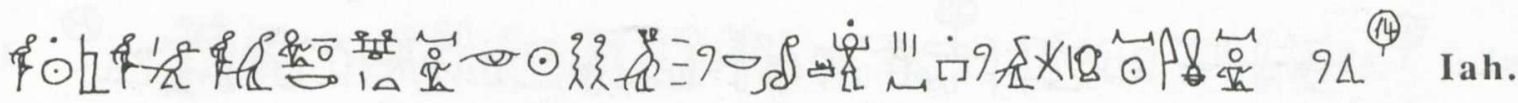

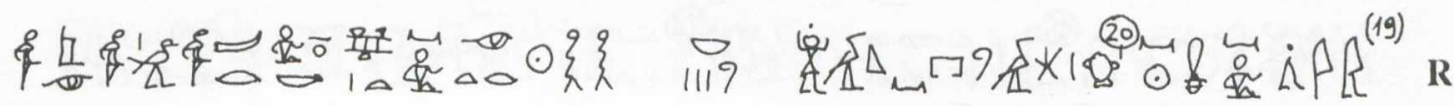

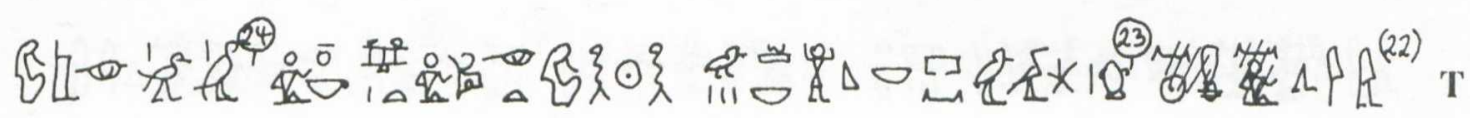

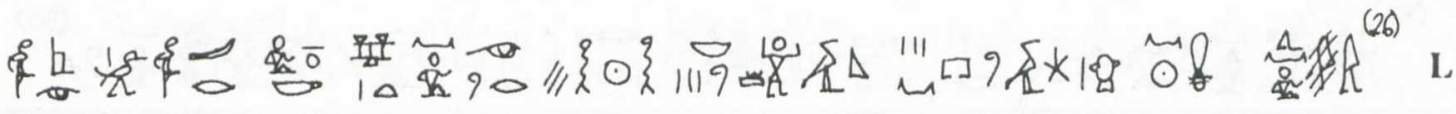

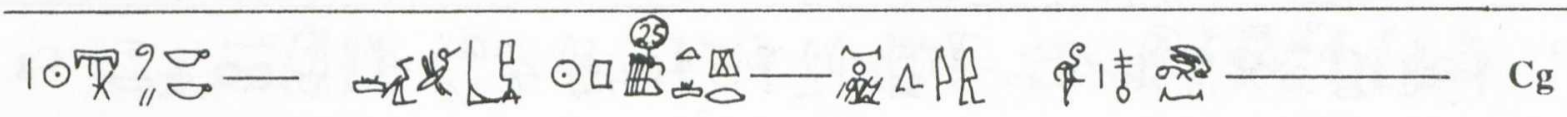

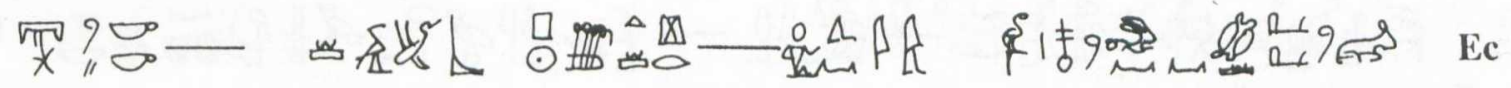

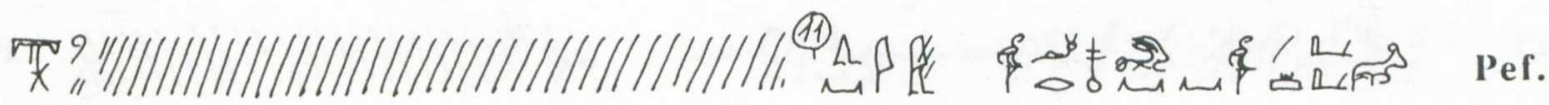
(13)

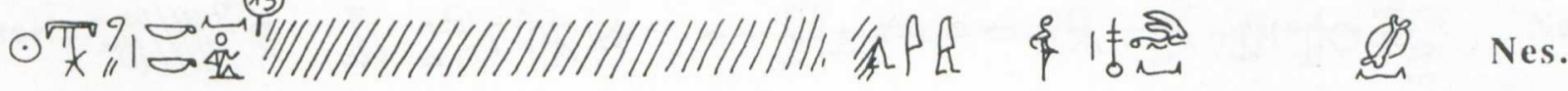

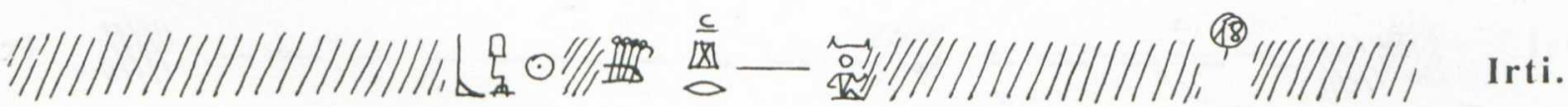

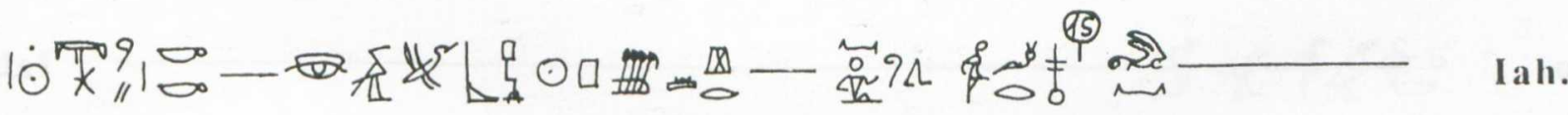

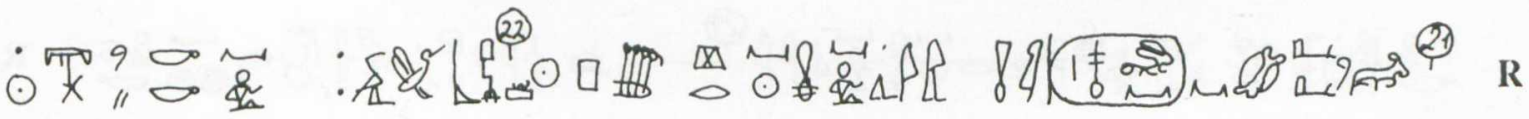

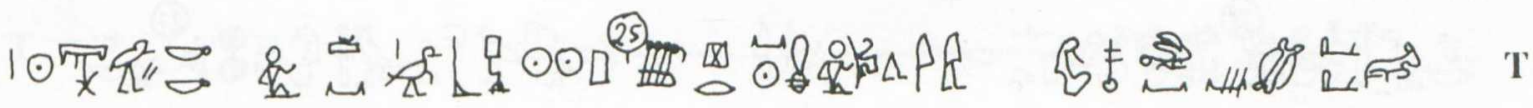

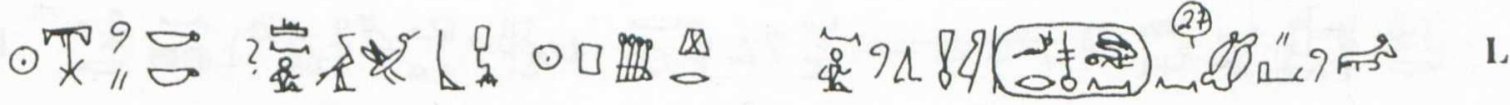
a D.-S: . 
186

URSULA VERHOEVEN

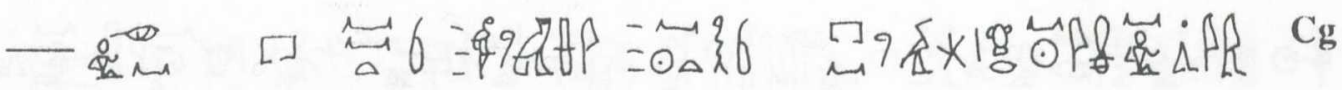

G

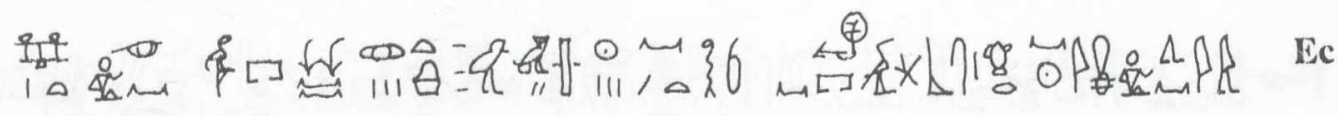

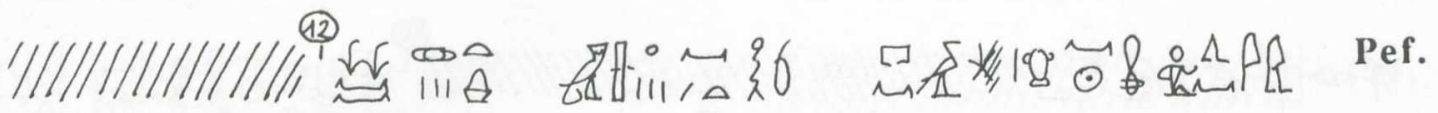

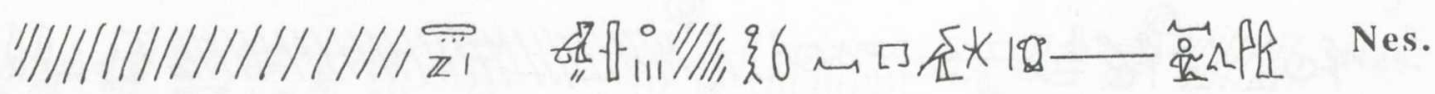
"I///I, - - - - - - - -

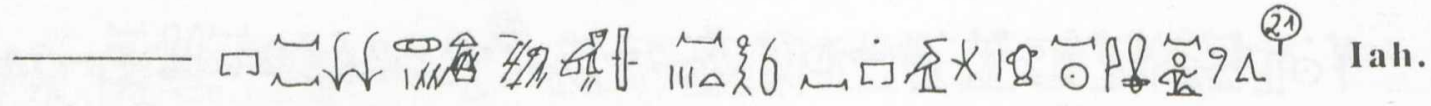

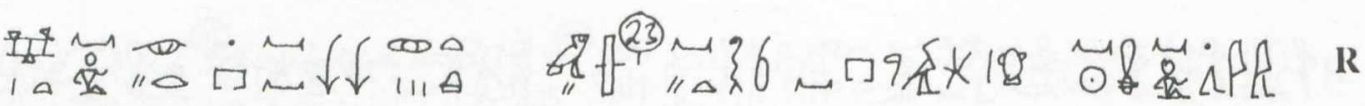

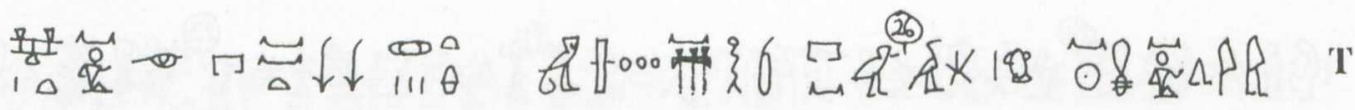

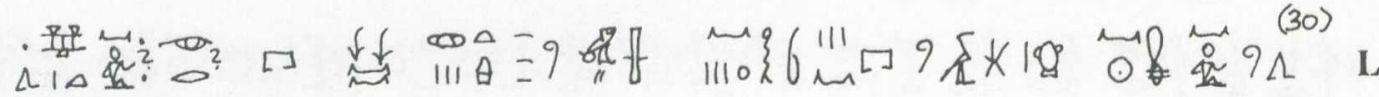

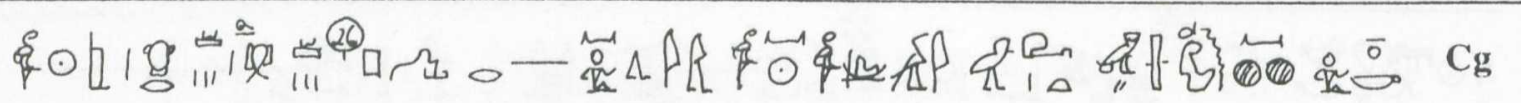

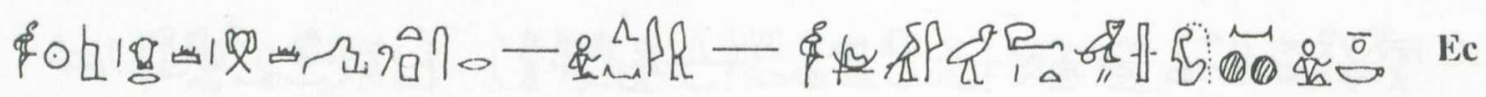

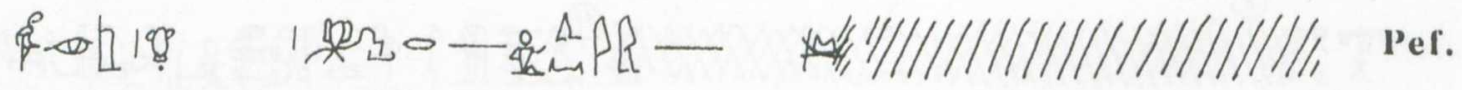

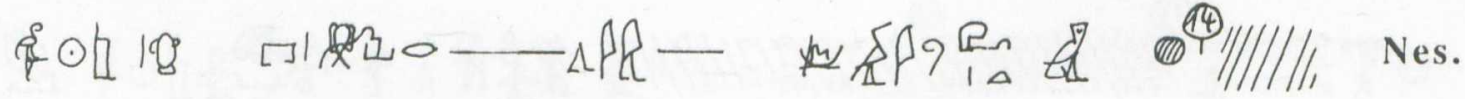
y///I, - . - . -

Iah.

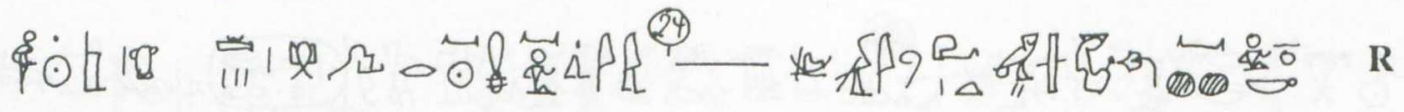
Ph-18

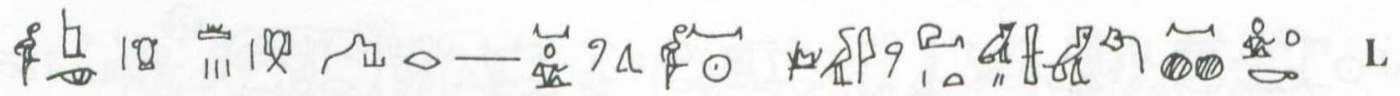

a Die gruppe III 103 ist nachtraghich wher die beile geschrieben worden. 
TOTENBUCHSPRUCH 146

187

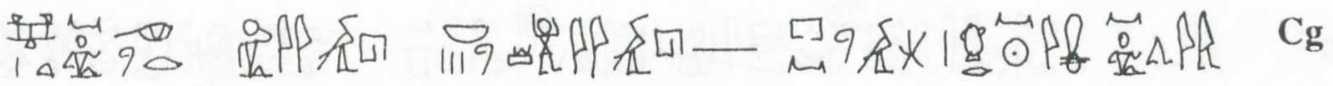

H

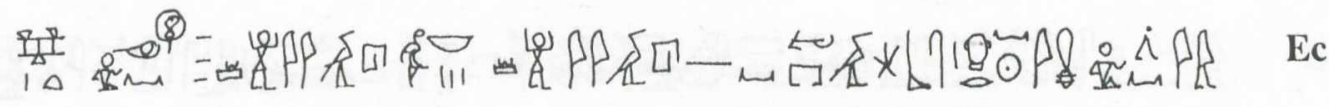

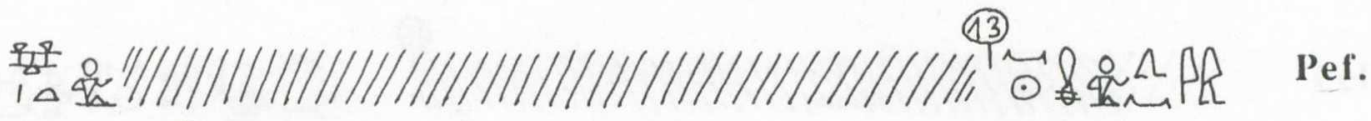

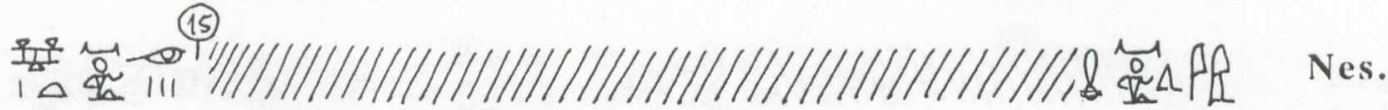

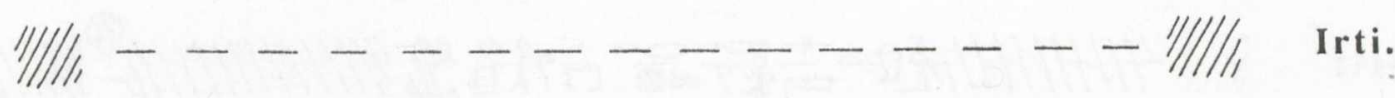

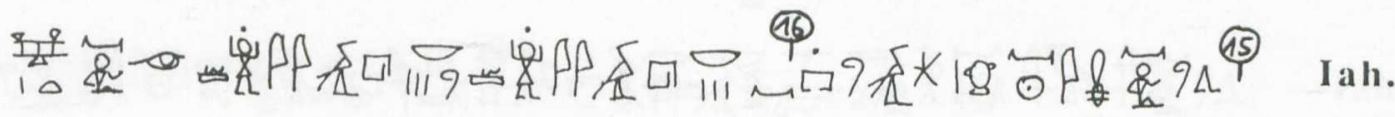

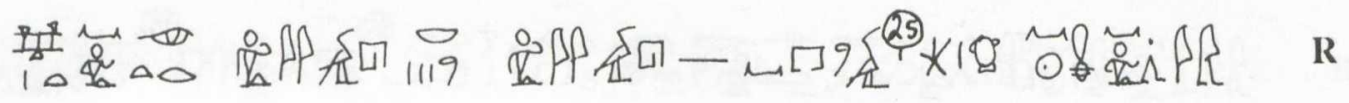

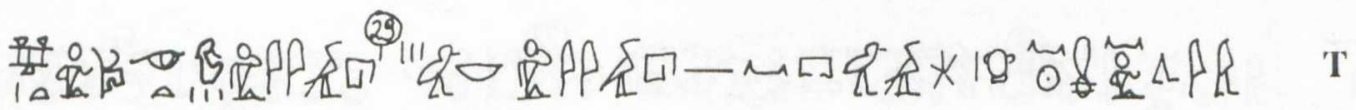

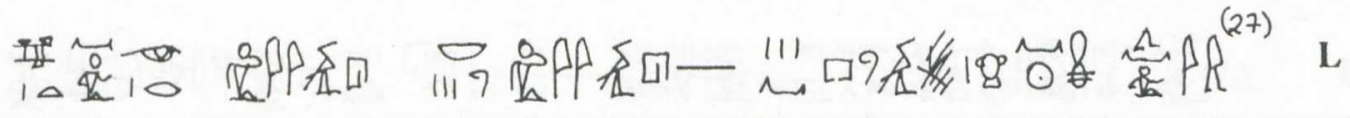

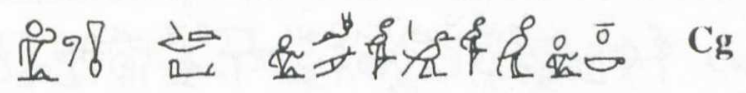

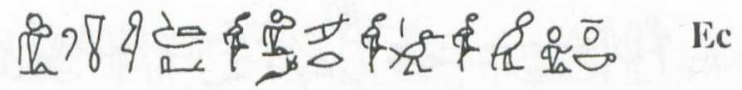

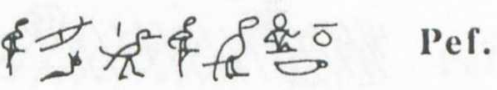

荄䘫一丝可 Nes.

"y/I/I/, - - - - - V///I, Irti.

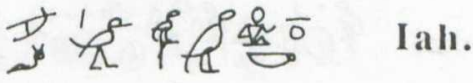

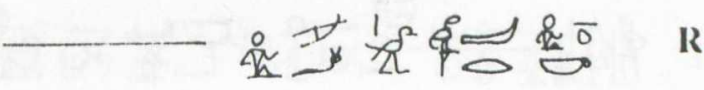

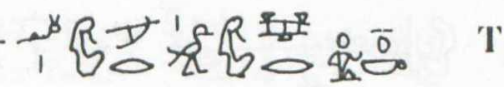

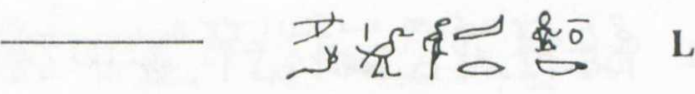




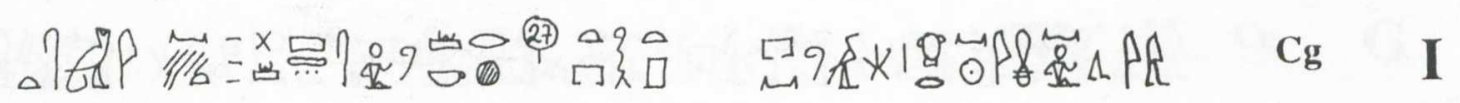

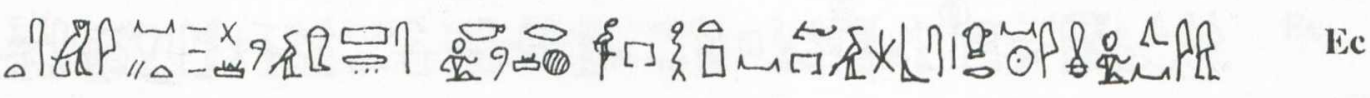

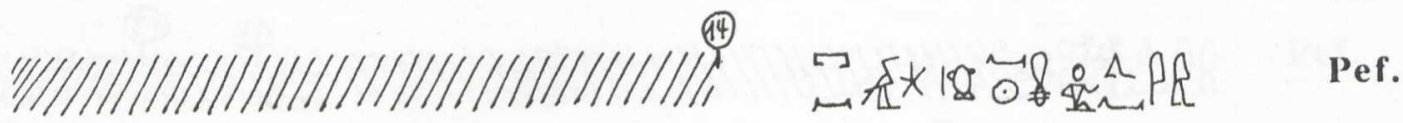

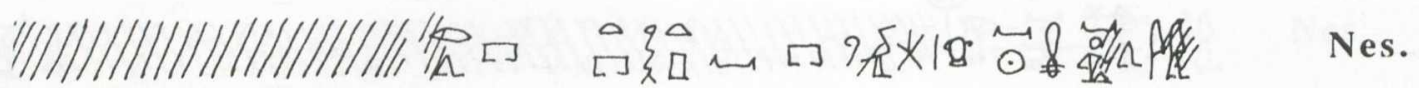

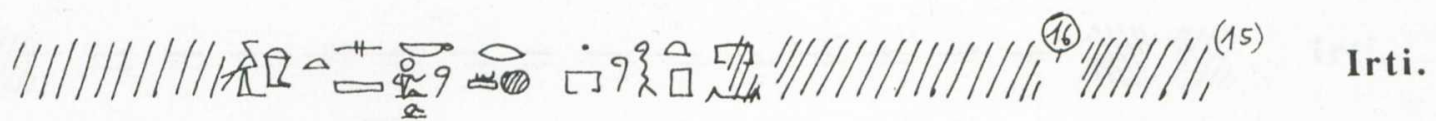

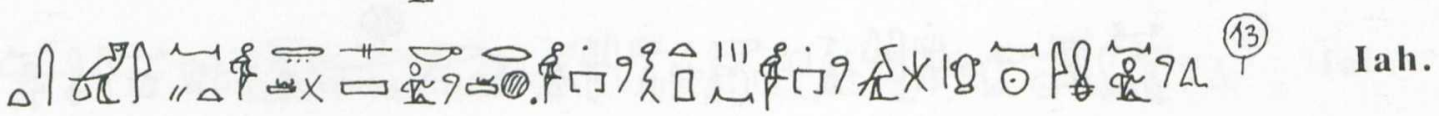

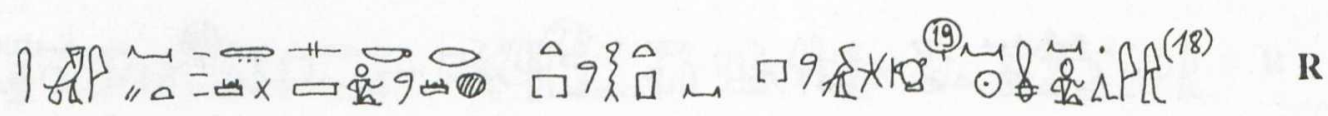

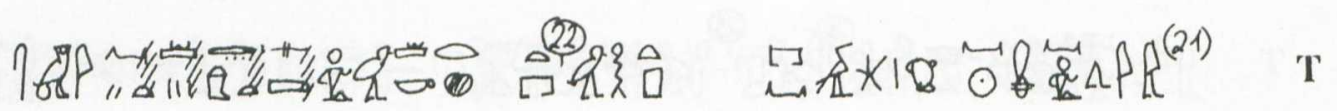

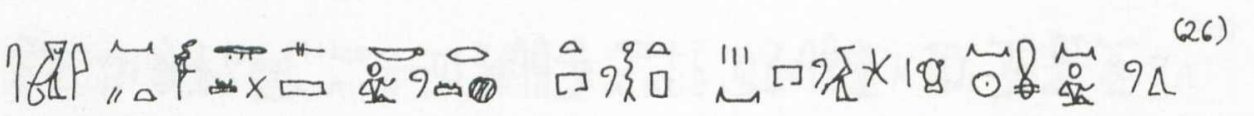

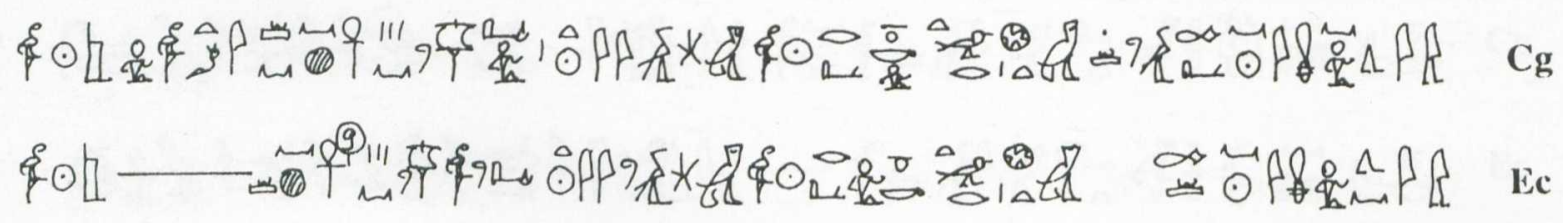

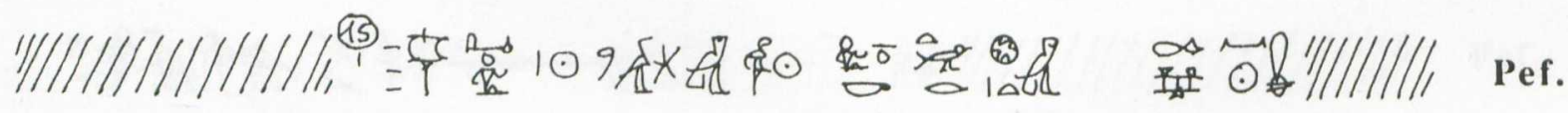

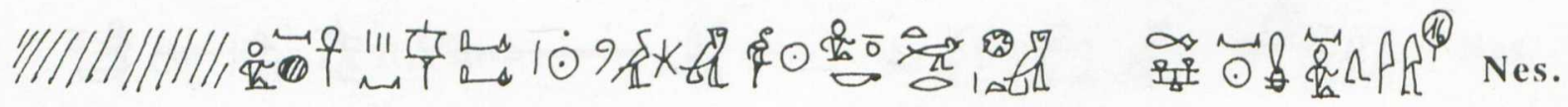

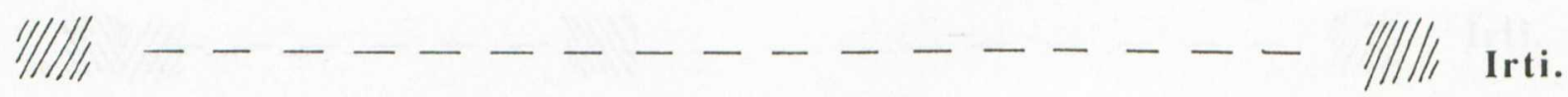

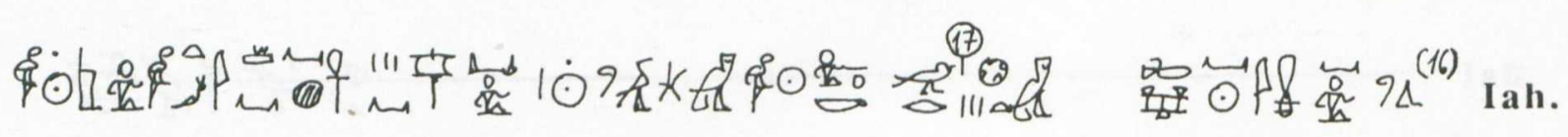

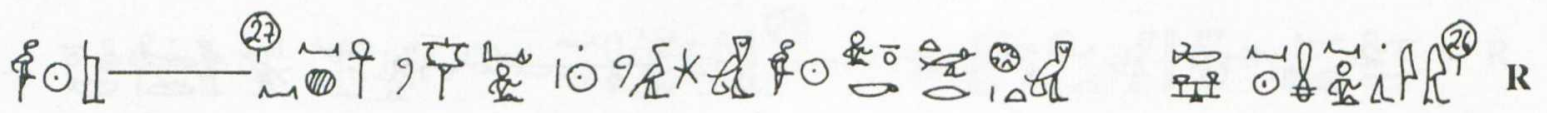

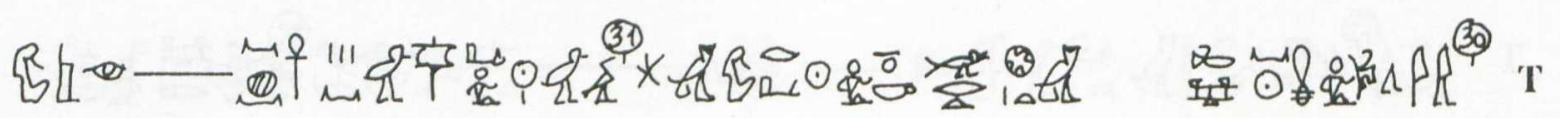

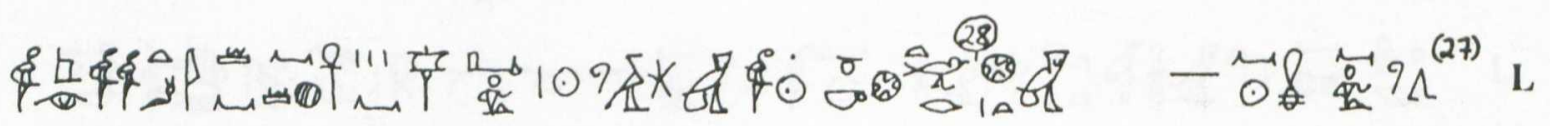
a D.S.: : 
TOTENBUCHSPRUCH 146

189

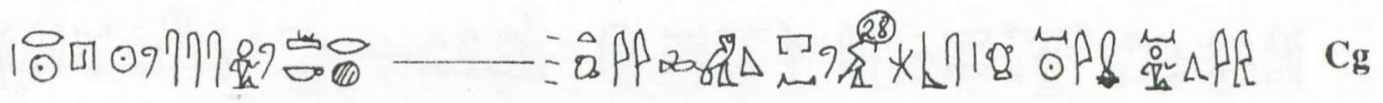

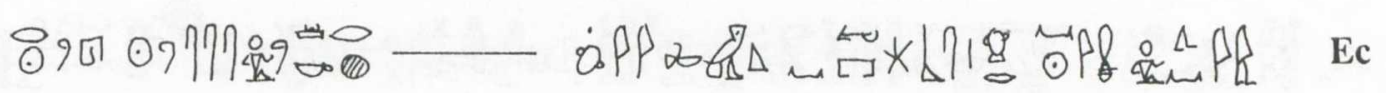
109प

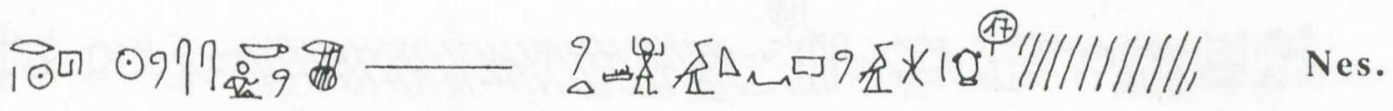

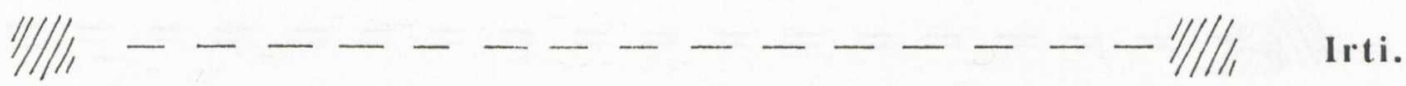

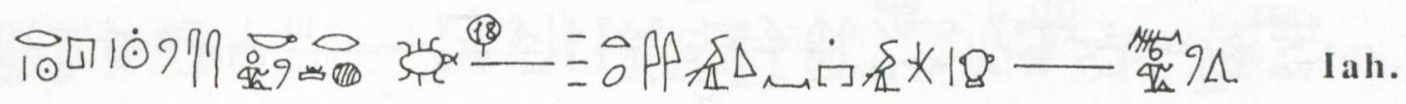

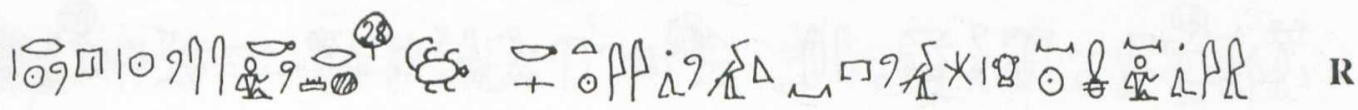

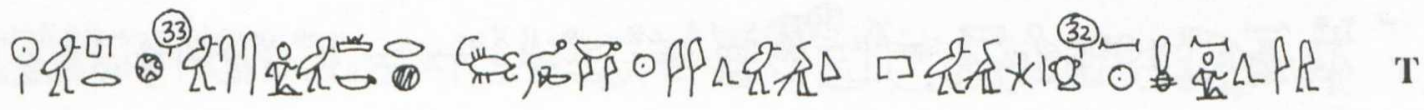

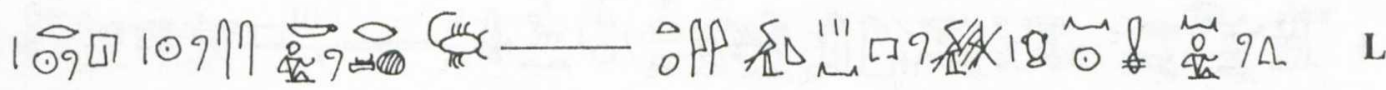

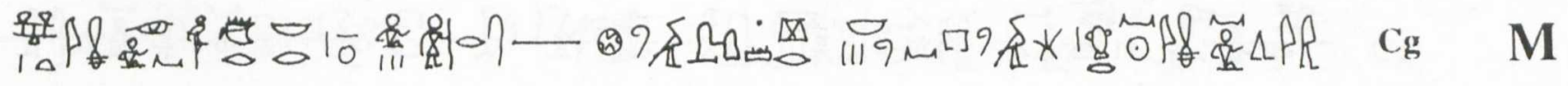

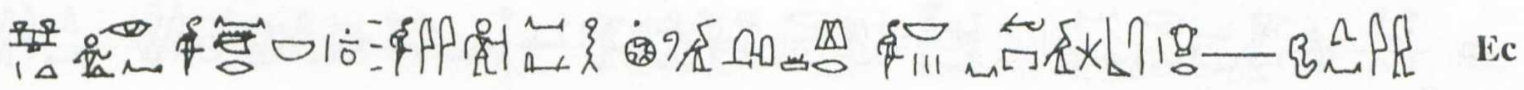

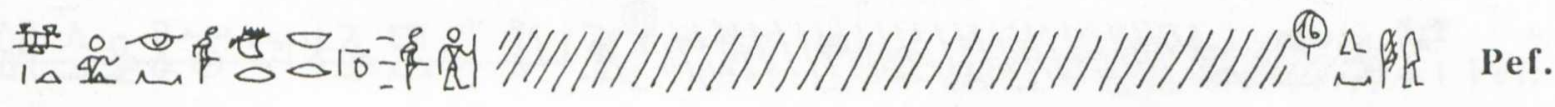

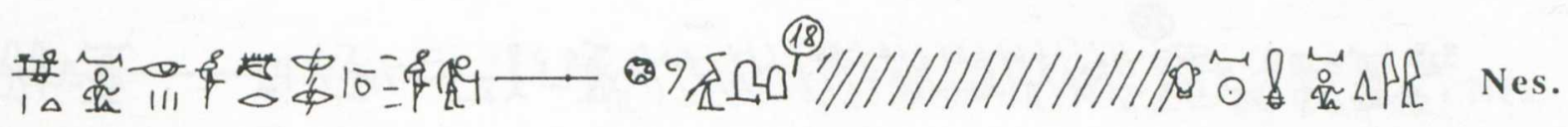
U//,

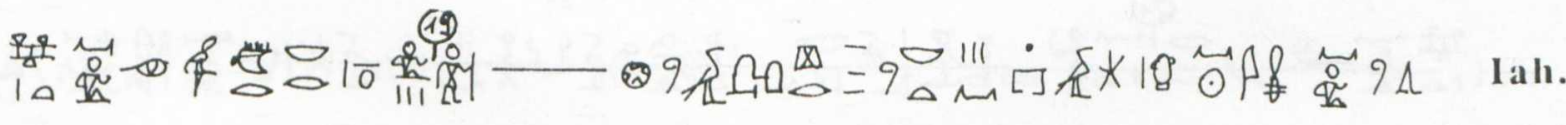

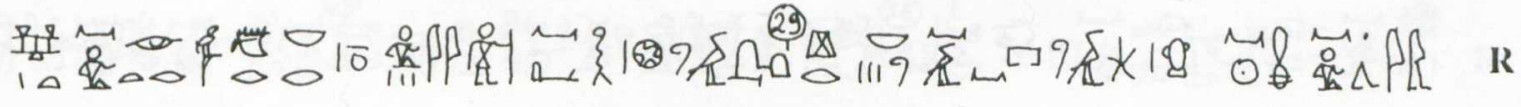

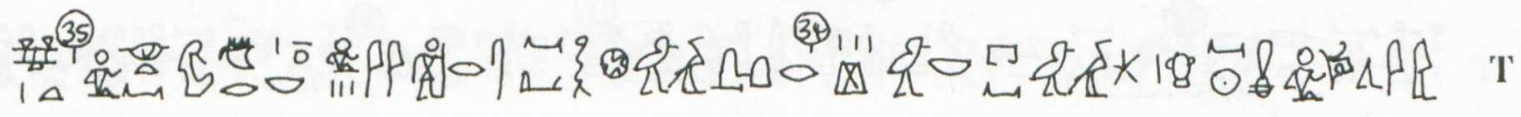

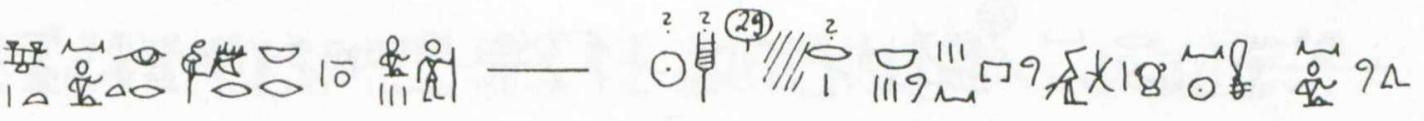
$\mathbf{L}$ 
190

URSULA VERHOEVEN

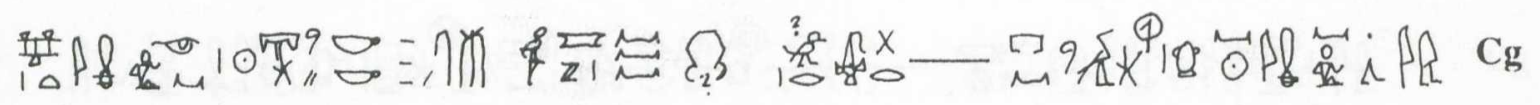

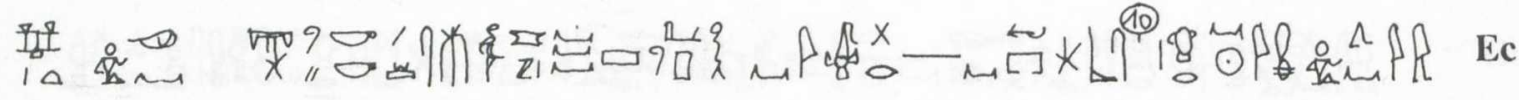

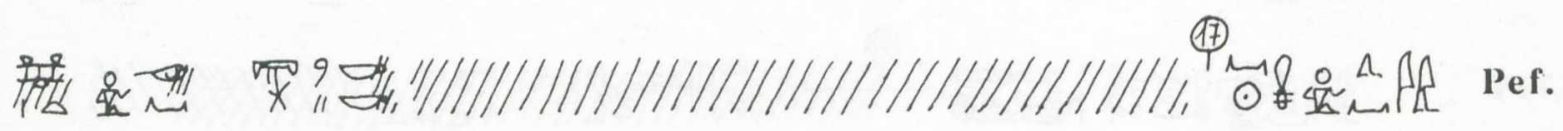

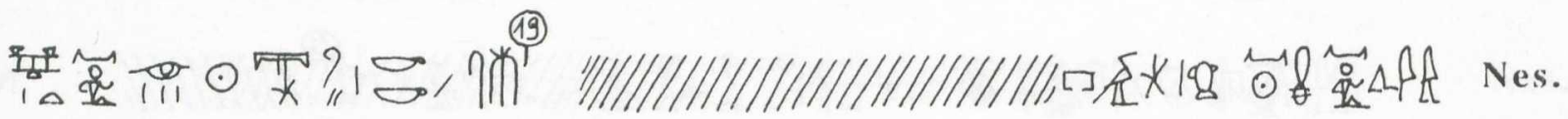
V////, - - - - - - - - - - -

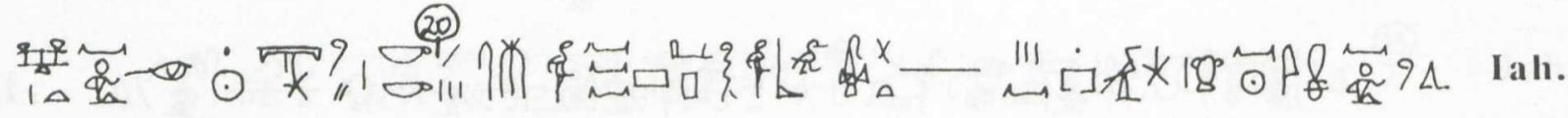

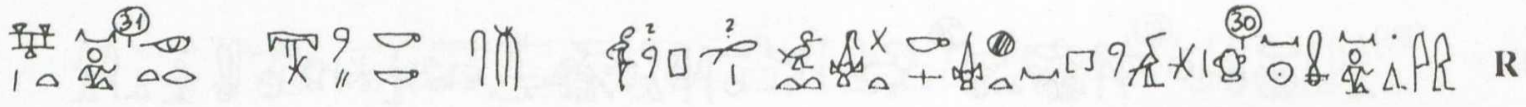

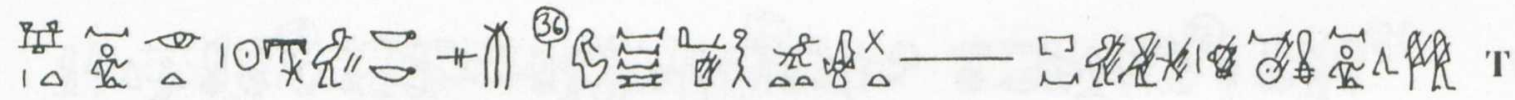

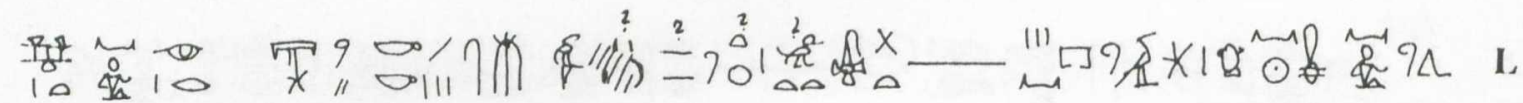

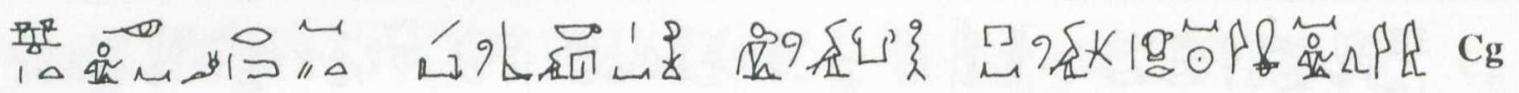
$\mathbf{O}$

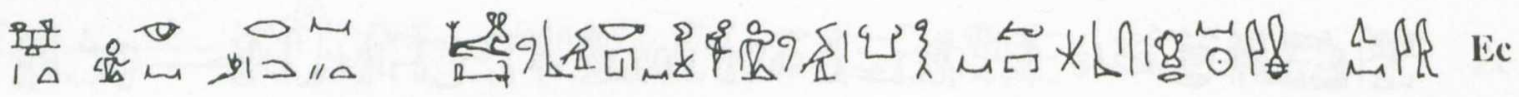

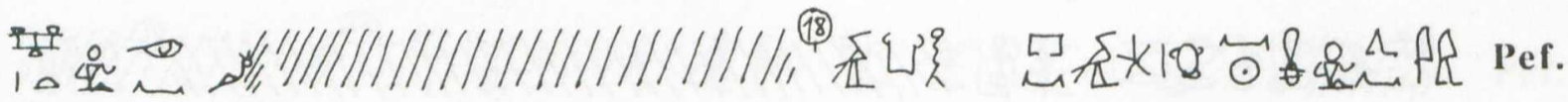

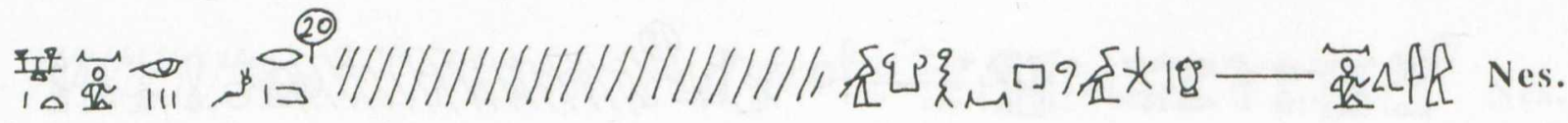
प//I, - - - - - - -

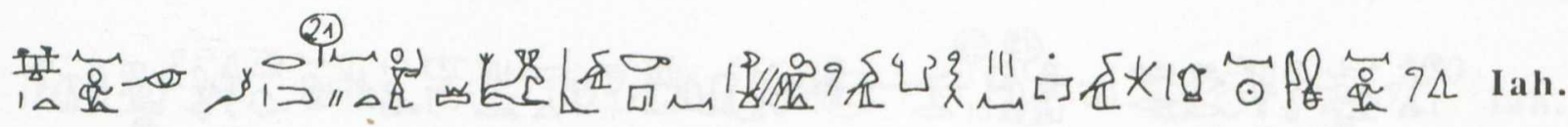

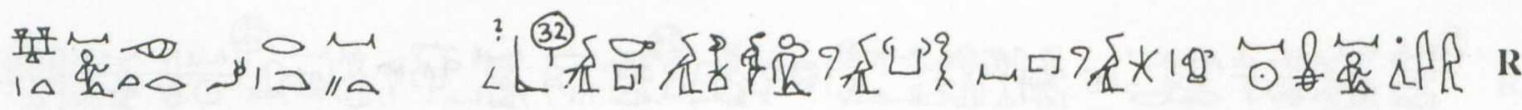

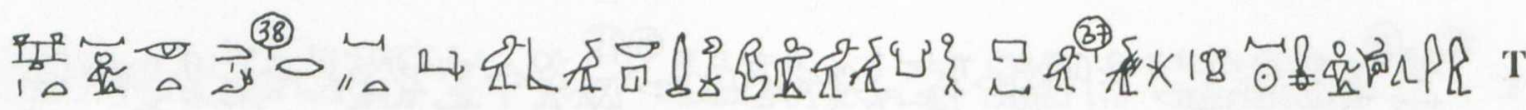

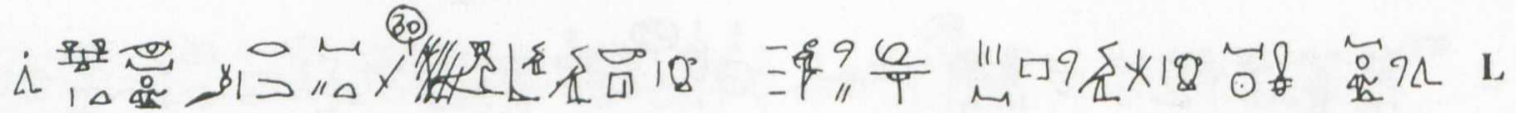




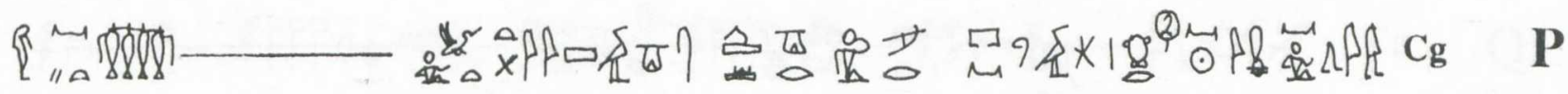

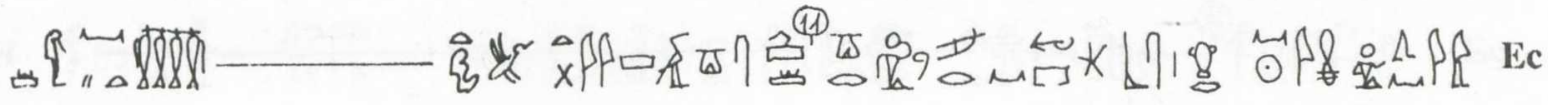

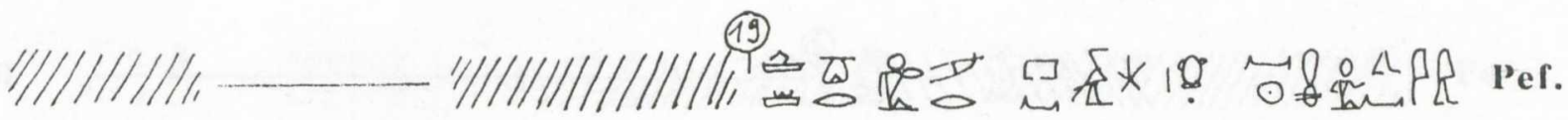
Q W//I, - - - - - - - - - - - - - W///, Irti.

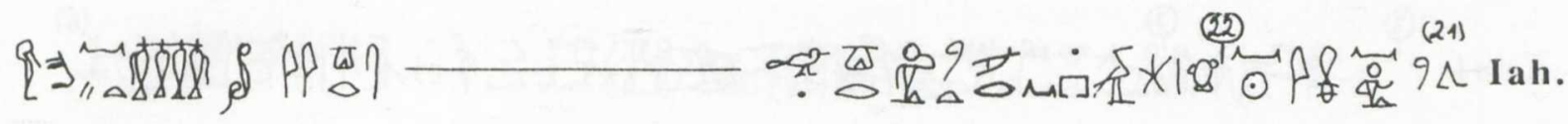
[व四"

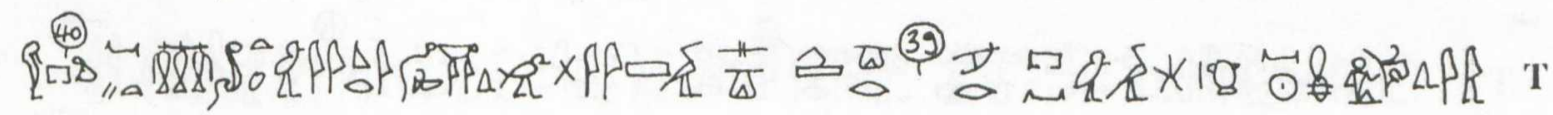

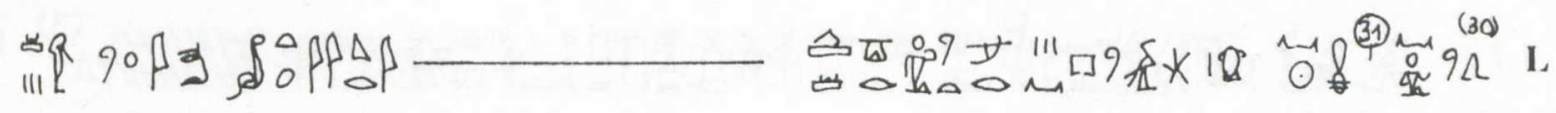
乎-

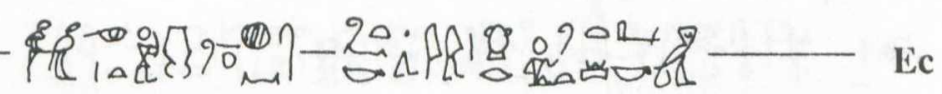

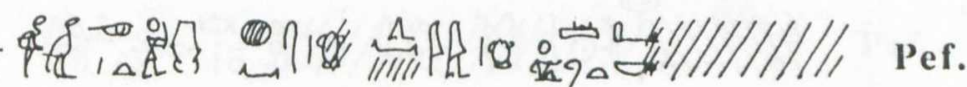

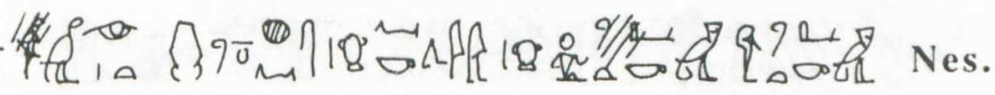

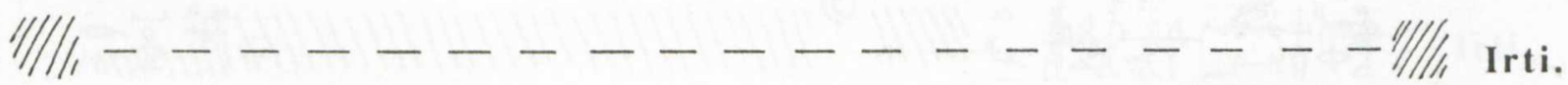

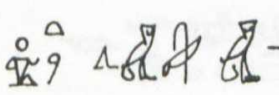
Iah.

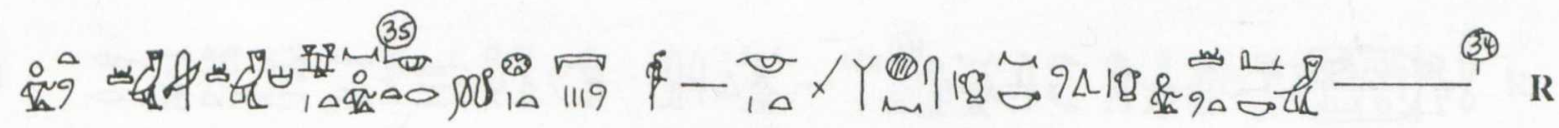

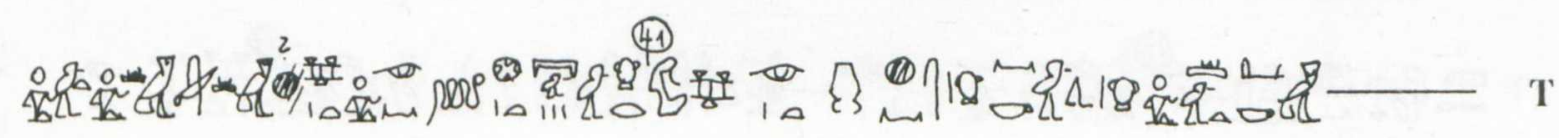

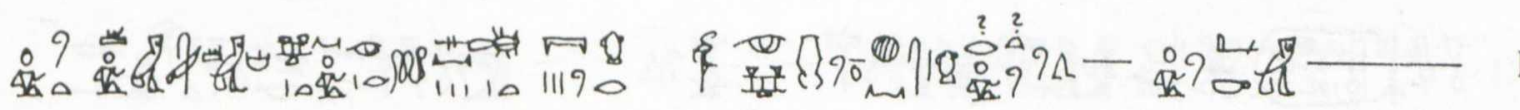
a Ein langes Spatium bis zum Ende der Zeile. 


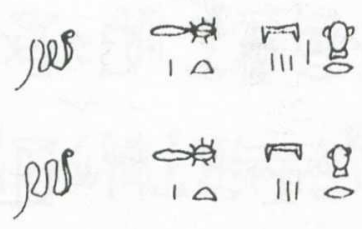

Pef.

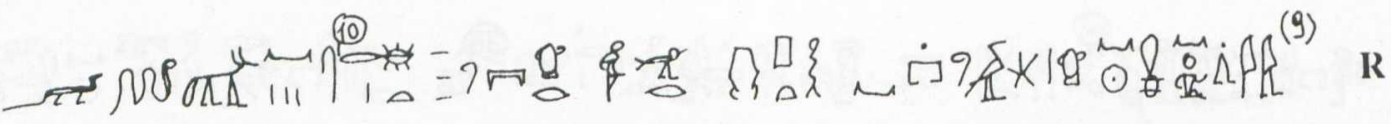

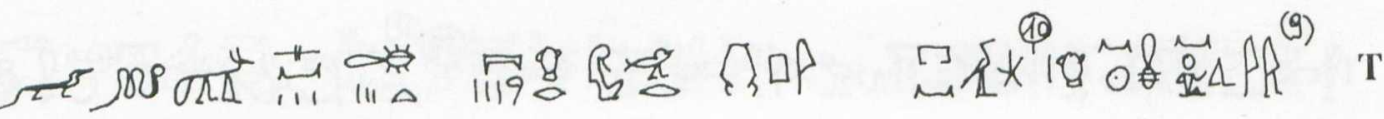

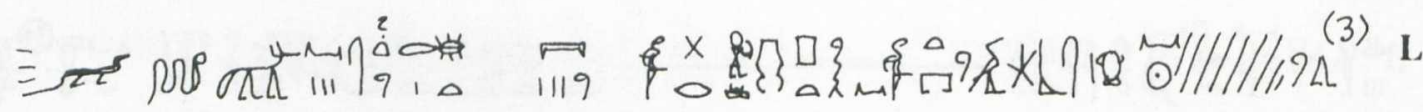

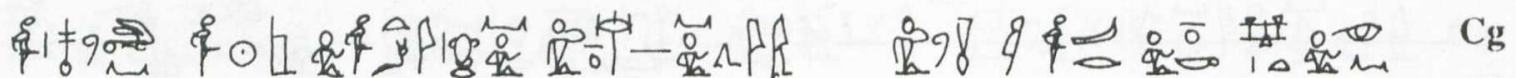

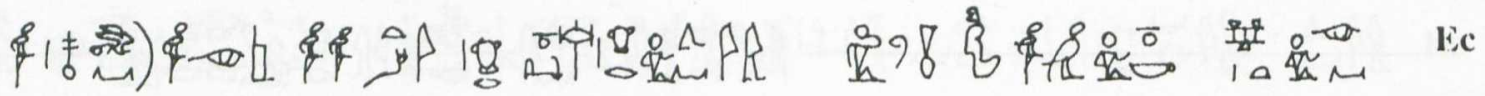

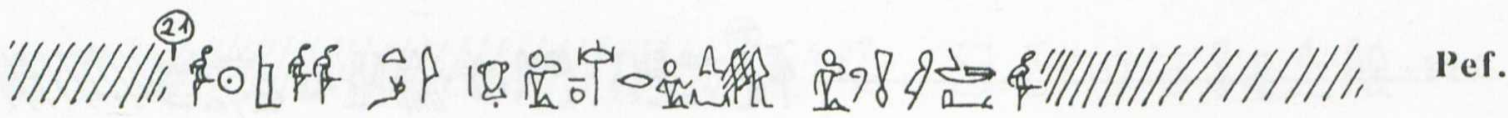

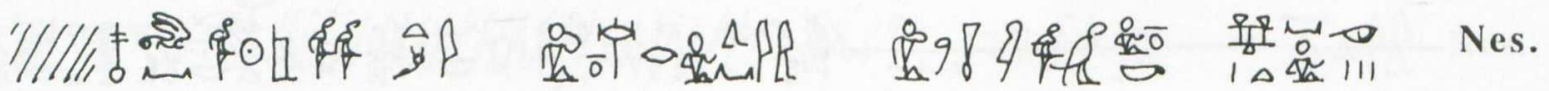

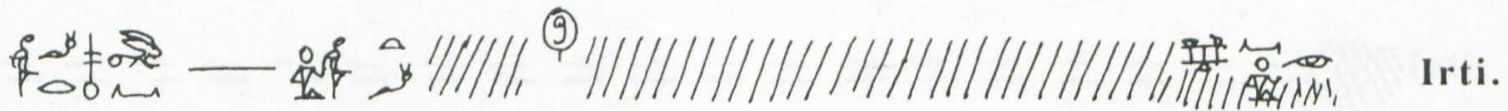

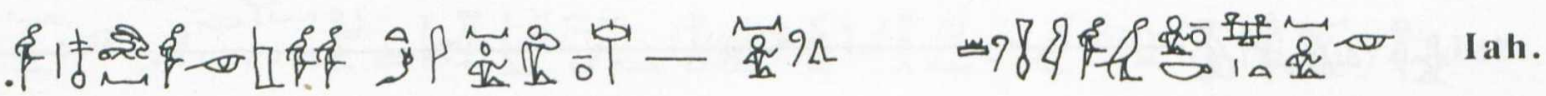

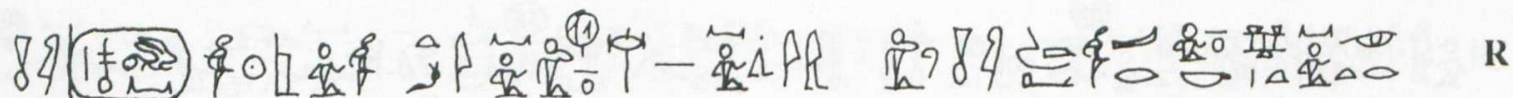

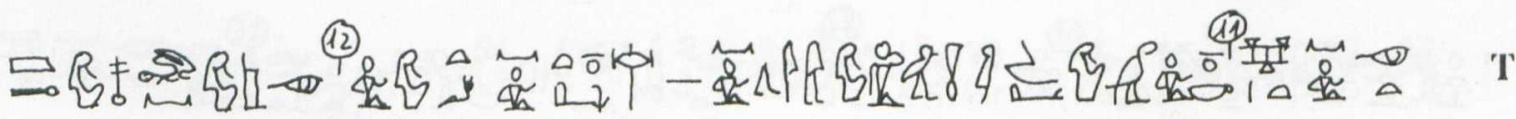

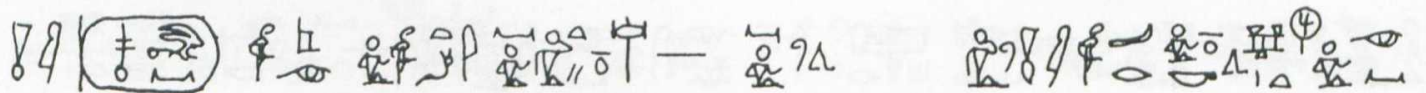




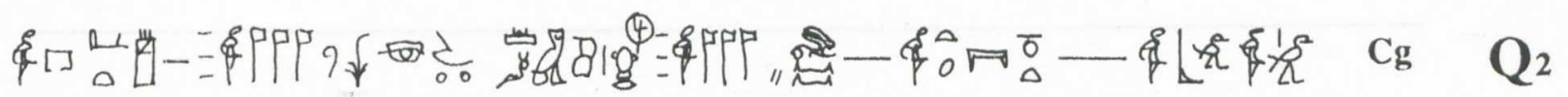

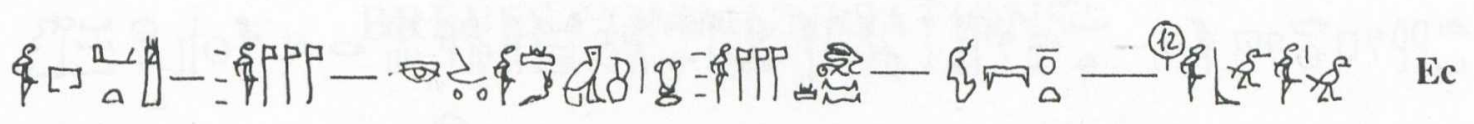

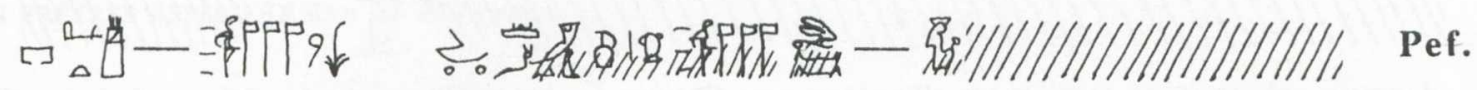

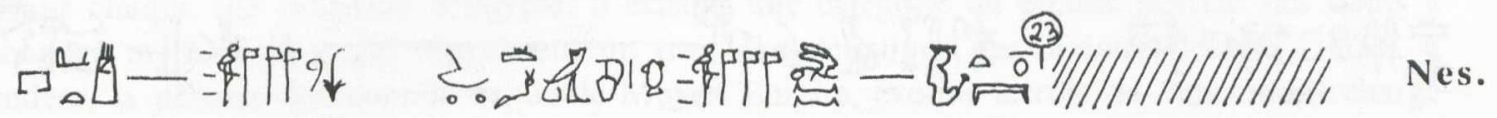

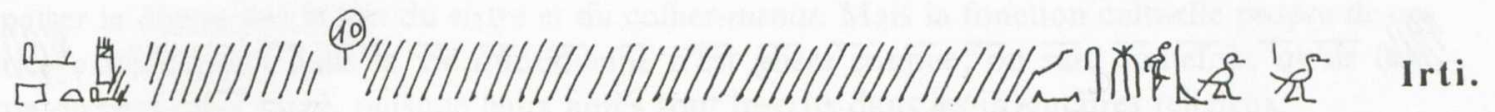

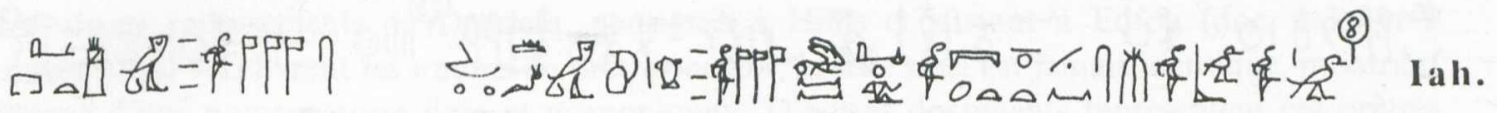

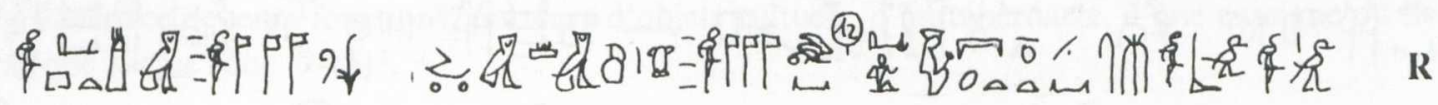

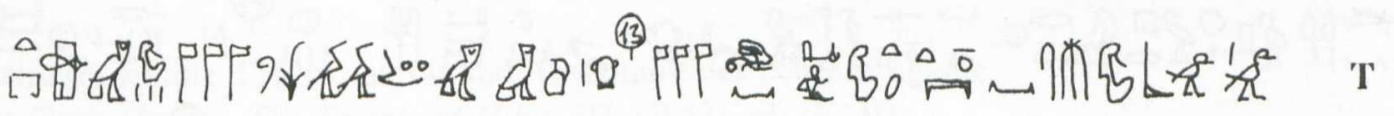

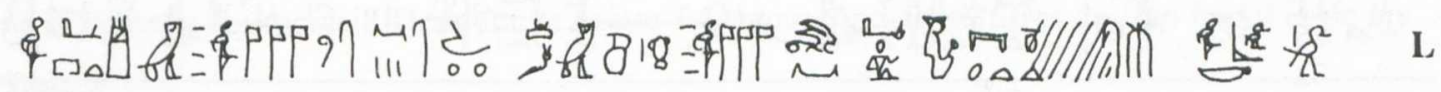

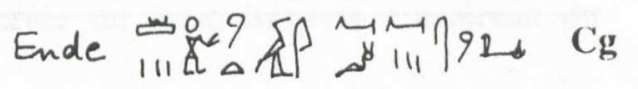

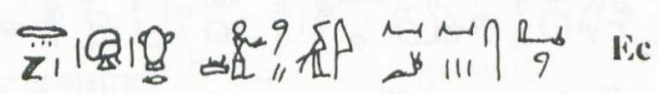

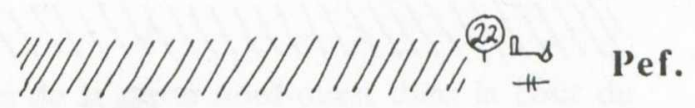

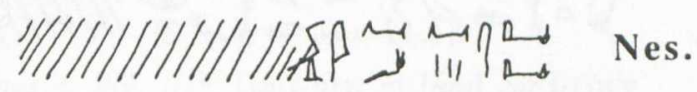

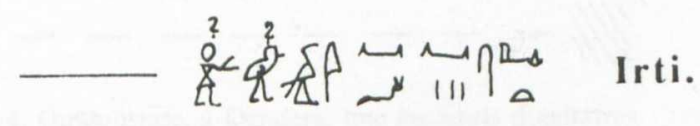

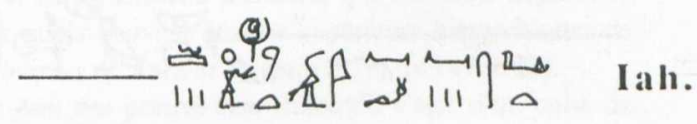

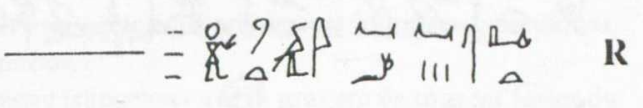

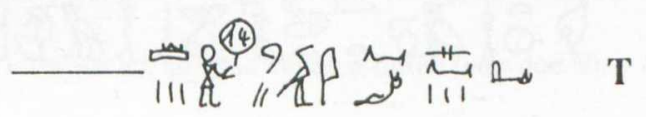

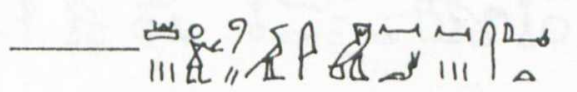




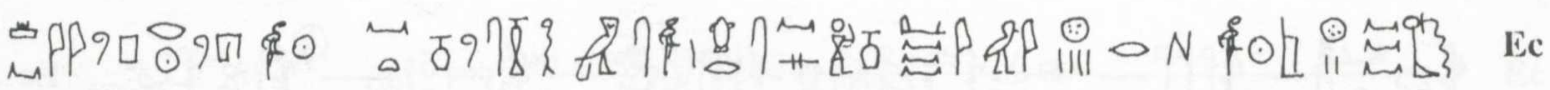

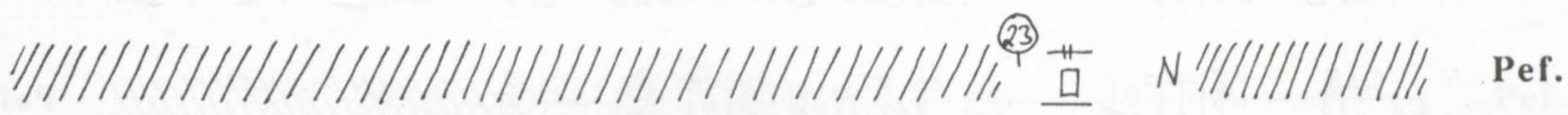

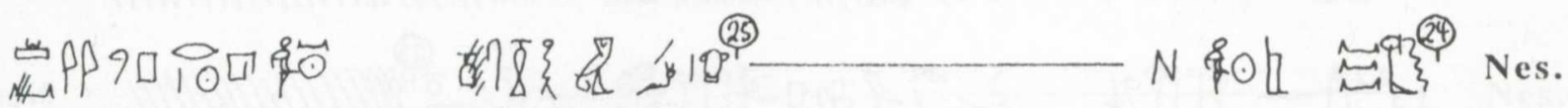
"I/I/I, - - - - - - - - W///, Irti.

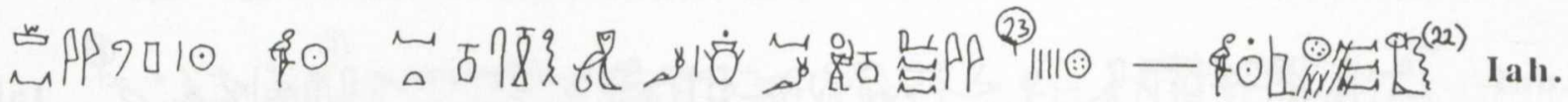

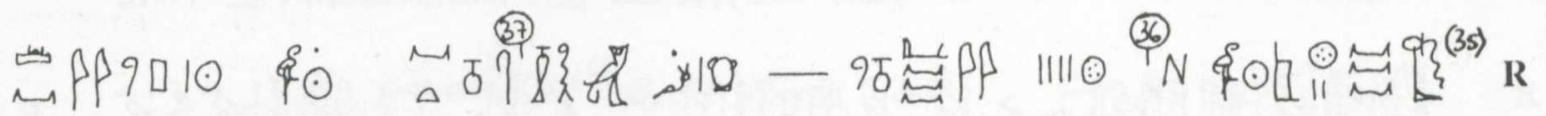

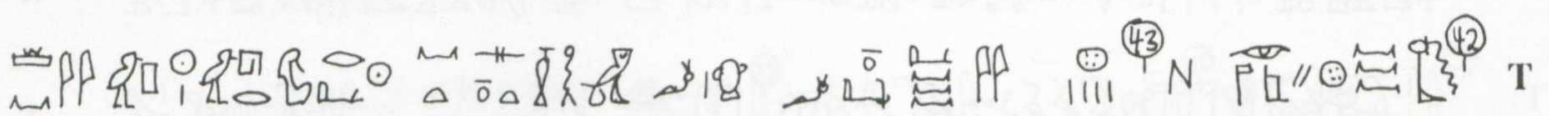

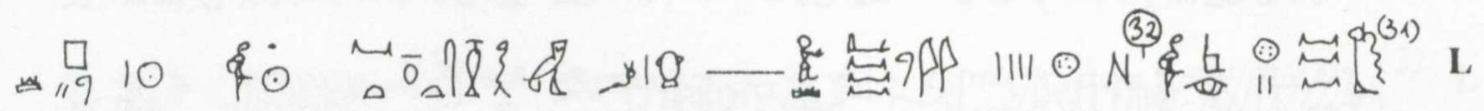

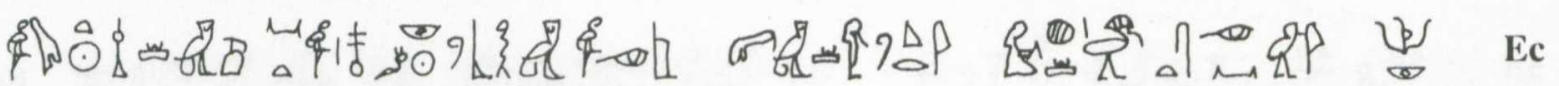

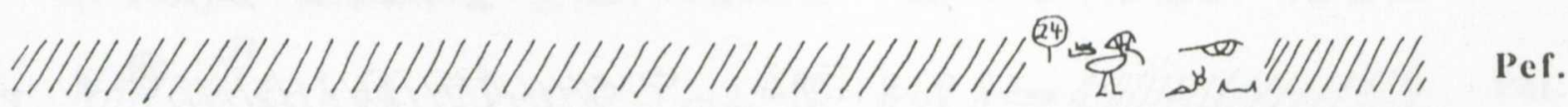

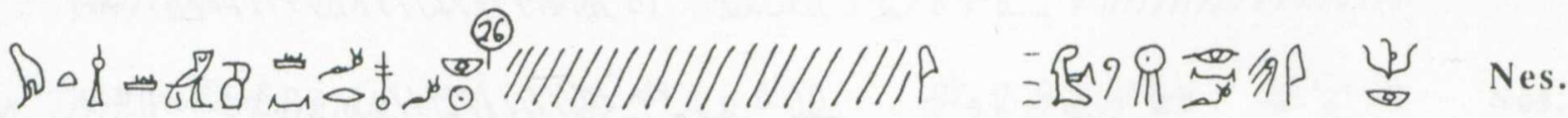

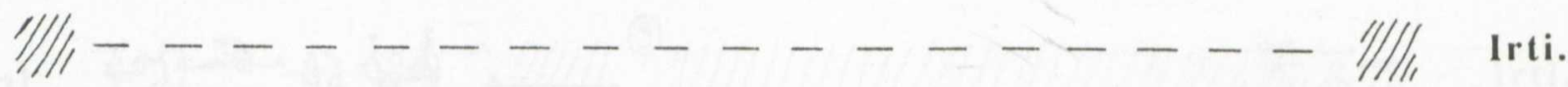
-

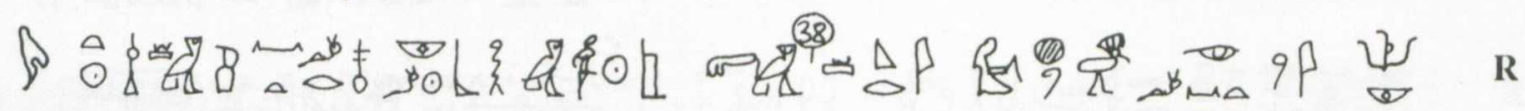

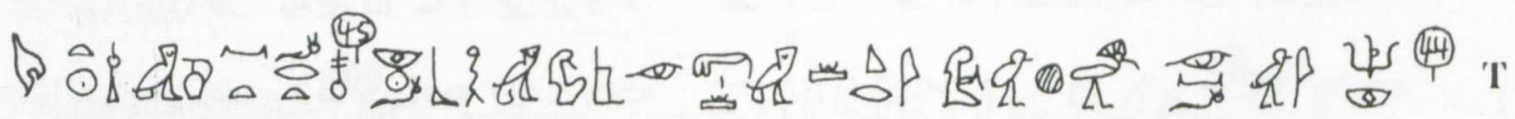

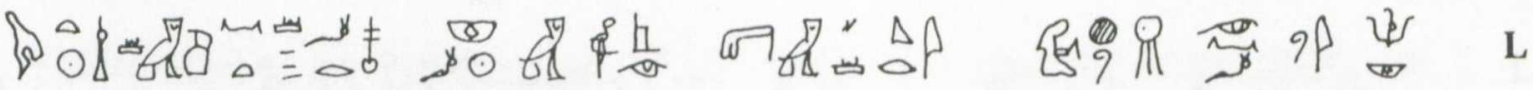

